\title{
Spatiotemporal analyses of cellular tractions describe subcellular effect of substrate stiffness and coating
}

Alicia Izquierdo-Álvarez ${ }^{1 *}$, Diego A. Vargas ${ }^{1 *}$, Álvaro Jorge-Peñas ${ }^{1}$, Ramesh Subramani $^{2}$, Marie-Mo Vaeyens ${ }^{1}$, and Hans Van Oosterwyck ${ }^{1,3+}$

1. Biomechanics Section, KU Leuven, Celestijnenlaan 300C, 3001 Leuven, Belgium

2. MeBioS, KU Leuven, Kasteelpark Arenberg 30, 3001 Leuven:/50

, Belgium

3. Prometheus, Division of Skeletal Tissue Engineering, KU Leuven, Herestraat 49, 3000 Leuven, Belgium

* Co-first author

+ Corresponding author

This is a pre-print of an article published in Annals of Biomedical Engineering. The final authenticated version is available online at: $h$ ttps://doi.org/10.1007/s10439-018-02164-2

\begin{abstract}
Cells interplay with their environment through mechanical and chemical interactions. To characterize this interplay, endothelial cells were cultured on polyacrylamide hydrogels of varying stiffness, coated with either fibronectin or collagen. We developed a novel analysis technique, complementary to traction force microscopy, to characterize the spatiotemporal evolution of cellular tractions: We identified subpopulations of tractions, termed traction foci, and tracked their magnitude and lifetime. Each focus consists of tractions associated with a local single peak of maximal traction. Individual foci were spread over a larger area in cells cultured on collagen relative to those on fibronectin and exerted higher tractions on stiffer hydrogels. We found that the trends with which forces increased with increasing hydrogel stiffness were different for foci and whole-cell measurements. These differences were explained by the number of foci and their average strength. While on fibronectin multiple short-lived weak foci contributed up to $30 \%$ to the total traction on hydrogels with intermediate stiffness, short-lived foci in such a number were not observed on collagen despite the higher tractions. Our approach allows for the use of existing traction force microscopy data to gain insight at the subcellular scale without molecular probes or spatial constraining of cellular tractions.
\end{abstract}

\section{KEY TERMS}

cell mechanics; traction force microscopy; endothelial cell; ECM protein 


\section{INTRODUCTION}

The formation of new blood vessels from preexisting ones, or angiogenesis, is of fundamental importance during development, growth and wound healing as well as for the development of tissue engineering strategies. Misregulation of angiogenesis is implicated in numerous diseases and pathological disorders including stroke, myocardial infarction, inflammatory diseases, or cancer (1-3). During angiogenesis, endothelial cells (ECS) interplay with their environment through chemical and mechanical interactions. Numerous studies about the biochemical regulation of angiogenesis have been performed (4-7). In contrast, studies that explore the mechanical regulation of angiogenesis remain scarce. In these, different 3D in vitro models of angiogenesis have been utilized to probe the effect of external mechanical loading and mechanical properties of the extracellular matrix (ECM) $(8,9)$. Depending on the hydrogel type and stiffness range probed, sprouting angiogenesis was found to increase with increasing hydrogel stiffness (10) or to peak at intermediate stiffness (11). As sprouting angiogenesis requires endothelial cell (EC) migration, which relies on mechanical interactions between ECs and their surrounding ECM, 2D single cell studies have been conducted in which EC-generated tractions are measured by means of Traction Force Microscopy (TFM) (12-15). TFM calculates cell tractions from measured displacements of the ECM (deformable hydrogel substrate) the cell is adhered to. TFM studies with ECs have explored the relation between cell area and exerted tractions (12), traction exertion in cells exposed to shear stress (13), traction exertion during cell spreading on a flat surface (14), and how the relation between cell area and traction changes from single cells to cell pairs (15). Overall, these studies found that there is a close relationship between substrate stiffness and traction generation.

More recently, high resolution TFM methods have been developed in order to study tractions at a subcellular level, more specifically at the level of single focal adhesions, given their importance in mechanotransduction (16-20). These methodologies have the drawback of requiring modifications to standard TFM protocols, such as the addition of fiducial markers of two different colors to ensure proper detection of fluorescent tags $(16,19)$, cellular transfection with molecular tags to visualize focal adhesion components $(20,21)$, or the use of super-resolution microscopy $(17,18)$.

With this work, we present a spatiotemporal analysis approach that can help to alleviate hurdles related to resolution and that are compatible with standard TFM protocols. In this approach, we zoom into the acquired TFM-based traction maps and track subsets of the tractions, which we term traction foci, on a scale closer to focal adhesion (FA) complexes.

\section{MATERIALS AND METHODS}

\section{Polyacrylamide hydrogel preparation and functionalization}

Polyacrylamide (PA) hydrogels were prepared as described by Tse et al. (22). To obtain the desired stiffness values $(1.4,2.7$, and $4.5 \mathrm{kPa})$, hydrogels were prepared by mixing different volumes of $40 \%$ acrylamide, $2 \%$ bis-acrylamide (Bio-Rad, Hercules, California) with distilled water. Stiffness of the gels was corroborated using Atomic Force Microscopy (AFM) (see Supplemental Materials for details). As fiducial markers for TFM, $200 \mathrm{~nm}$ carboxylate-modified microspheres (FluoSpheres ${ }^{\circledR}$, ThermoFisher Scientific, Waltham, MA) were added to the mixture at a 1:60 ratio.

Hydrogels were functionalized with either $5 \mu \mathrm{g} / \mathrm{ml}$ of human fibronectin (Sigma-Aldrich, St. Louis, MO) or $100 \mu \mathrm{g} / \mathrm{ml}$ rat tail collagen I (BD bioscience, San Jose, CA). Even though the ECM protein concentrations 
used are different, in both situations the gels are saturated with the ECM protein, meaning the entire surface of the gel is covered by the coating molecule without any gaps of the order of magnitude of the traction foci (Figure S1 in Supplemental Materials). For a more detailed description of the hydrogel preparation and functionalization processes, see Supplemental Materials.

\section{Cell culture}

Green fluorescence protein (GFP)-expressing human umbilical vein endothelial cells (HUVECs) (Angioproteomie, Boston, MA), were seeded at low density on polyacrylamide hydrogels with desired stiffness. Cells were allowed to attach and spread overnight before imaging. For a more detailed description of the culture setup, see Supplemental Materials.

\section{Live cell imaging and cell mask extraction}

Live imaging was done using an Olympus FluoView ${ }^{\mathrm{TM}}$ FV1000-IX81(Olympus, Tokio, Japan) confocal microscope. Images of the cells and the stressed hydrogels (deformed under cellular tractions) were taken simultaneously every $2.25 \mathrm{~min}$ for approximately $1 \mathrm{~h}$ (67.5 min or 30 time-points).

Acquired cell images were enhanced by a penalized least squares-based denoising step (23), followed by a contrast stretching operation to highlight cells from the image background. Then, a multi-level isodata thresholding algorithm was used to segment cell bodies at each time step and to obtain the cell mask.

\section{Dynamic Traction Force Microscopy}

For each time point, substrate displacements with respect to the relaxed state were measured, and corresponding tractions were calculated. Cellular tractions were recovered from the computed displacements by Tikhonov regularized Fourier Transform Traction Cytometry algorithm (16), which was modified to avoid the unwanted effects of the Young's modulus of the substrate in the regularization parameter. See Supplemental Materials for detailed information on displacement field calculation, traction recovery, and proposed corrections. Video S1 in the Supplemental Materials shows a representative example of the effects of both non-corrected and corrected regularization applied to the same displacement field.

\section{Traction footprint}

We chose the Fourier Transform Traction Cytometry (FTTC) algorithm (24) to recover cellular tractions. Relative to methods that recover tractions in the spatial domain, recovering traction in the Fourier domain is computationally more efficient; however, the spatial regions where tractions are exerted cannot be specified a priori (25). As an alternative constrained methods, all in the spatial domain, have been developed, such as the Boundary Element Method, Finite Element Method, and Traction Reconstruction with Point Forces (TRPF) (26-28). We have used an unconstrained FTTC algorithm in which it is assumed that tractions can be localized at every position within the field of view (FOV). This is appropriate for our experimental setup because it is difficult to determine with certainty where the cell boundary is located; this difficulty arises from variation of fluorescence from cell to cell and low fluorescence at very thin protrusions and lamellipodia. With FTTC, it is the method itself which determines where tractions are exerted or not, indicated by either large traction value or a negligible value calculated at each position 
within the FOV, respectively. How negligible this value is depends on the experimental setup, which always entails a certain amount of experimental error, producing a small background level (Figure 3a).

To account for this background value generated by unsconstrained FTTC, we defined a region of interest to distinguish the cell contribution from the background values inherent to experimental errors and computational methods: the traction footprint (see Figure $3 a$ for an example). This region was automatically obtained by applying Otsu's thresholding algorithm to the magnitude of the recovered tractions.

\section{Global traction metrics}

These following metrics were calculated using the entire traction footprint:

Force dipoles $\left(M_{i j}\right)$ :

The dipoles reveal the principal directions of the cellular tractions, their eccentricity (i.e. deviation from circular distribution around cell mask centroid), and their contractile or tensile nature. The force dipoles are calculated by obtaining the first-order moments of the force (Equation 1)(29).

$$
M_{i j}=\sum_{p=1}^{P_{s}} x_{i, p} t_{j, p} \Delta_{x y}^{2}
$$

$\boldsymbol{M}$ is a $2 \times 2$ tensor with components $M_{i j}$, where $i$ and $j$ refer to the coordinate axes $(x=1, y=2) . x_{i, p}$ is the ith component of the position of each pixel $(p)$ from the origin, defined as the centroid of the cell mask. $t_{j, p}$ is the $j$ th component of the traction at the corresponding pixel $(p) . \Delta_{x y}^{2}$ is the area of each pixel. To calculate $\boldsymbol{M}$, the integral is approximated as the discrete sum over the pixels that constitute the traction footprint $\left(P_{S}\right)$.

The diagonal components of the tensor describe the contractile/tensile forces along the axes $\left(M_{x x}\right.$ and $M_{y y}$ ), and the off-diagonal components describe the torque with respect to an axis (i.e. total torque given by $\left.M_{x y}-M_{y x}\right)$. By diagonalizing $\boldsymbol{M}$, the force distribution is reduced to two dipoles; these are the two principal directions of stress (eigenvector). The dominant orientation (major dipole) is the eigenvector corresponding to the largest eigenvalue. The minor dipole is the eigenvector corresponding to the smaller eigenvalue. The eigenvalues are signed quantities; the sign indicates whether the dipole is contractile (negative sign) or tensile (positive sign). The dipole ratio is the quantity obtained by dividing the magnitude of the major dipole by the magnitude of the minor dipole and is a measure of force polarization of the cell. Figure S2 in the Supplemental Materials shows a sample calculation of the force dipole for one of our cells.

Total force magnitude:

$$
|\boldsymbol{f}|_{\text {tot }}=\sum_{p=1}^{P_{s}} \sqrt{\left(t_{x}^{2}+t_{y}^{2}\right)_{p}} \Delta_{x y}^{2}
$$

Average traction magnitude: 


$$
|\boldsymbol{t}|_{\text {avg }}=\frac{|\boldsymbol{f}|_{\text {tot }}}{A_{\text {cell }}}
$$

Where $A_{\text {cell }}$ represents the area of the cell mask.

Maximum traction magnitude:

$$
|\boldsymbol{t}|_{\max }=\max _{p \in P_{s}}\left(\sqrt{t_{x}^{2}+t_{y}^{2}}\right)_{p}
$$

\section{Local traction analysis}

The traction footprint was further segmented into traction foci (sample segmentation in Figure 3a). Segmentation was performed by applying a watershed transformation on the traction footprint, using local maxima in traction magnitude (peak traction magnitude values) as seeds. Identification of this local peak was done using the imregionalmax function in MATLAB (Mathworks, Natick, MA, USA), which identifies the local maxima in a grayscale image (i.e. traction magnitude map). This meant that per peak, a single focus was defined.

Individual foci were tracked in time: Foci identified in consecutive time-points were identified as being one and the same based on spatial overlap. More information on this algorithm can be found in the Supplemental Materials. Once individual foci were tracked, they were characterized by calculating a series of features at each time-point:

Area:

$$
A_{\text {foc }}=P_{f o c} \Delta_{x y}^{2}
$$

$P_{f o c}$ denotes the number of pixels that make up a focus. $\Delta_{x y}^{2}$ is the area of each pixel.

Force:

$$
F_{\text {foc }}=\sum_{p=1}^{P_{f o c}}\left(\sqrt{t_{x}^{2}+t_{y}^{2}}\right)_{p} \Delta_{x y}^{2}
$$

$t_{x}$ and $t_{y}$ are the $x$ and $y$ components of the traction vector $(\boldsymbol{t})$ calculated for each pixel $(p)$.

\section{Mean Focus Traction:}

$$
|\boldsymbol{t}|_{\mathrm{foc}}=\frac{F_{\mathrm{foc}}}{A_{\mathrm{foc}}}=\frac{\sum_{p=1}^{P_{f o c}}\left(\sqrt{t_{x}^{2}+t_{y}^{2}}\right)_{p}}{P_{f o c}}
$$


To compare across conditions, these features were then averaged in time for each time-point in which a given focus was identified. This single value per focus was then averaged for all foci in each experimental condition.

Focus Lifetime:

The number of traction foci that were detected for each cell throughout the experiment duration and their individual lifetimes $(\lambda)$ were also measured.

Percentage (\%) total force:

The contribution of foci with a particular lifetime to the total force magnitude exerted by a single cell:

$$
(\% F)_{\lambda}=\frac{\left\langle F_{\text {foc }}\right\rangle_{\lambda}\left\langle N_{\text {foc }}\right\rangle_{\lambda}}{\sum_{\lambda=1}^{\lambda_{\max }}\left\langle F_{\text {foc }}\right\rangle_{\lambda}\left\langle N_{\text {foc }}\right\rangle_{\lambda}}
$$

$\left\langle F_{\text {foc }}\right\rangle_{\lambda}$ denotes the average force exerted by all foci with a lifetime $\lambda ; \lambda$, corresponds to acquisition steps and thus ranges from 1 to $\lambda_{\max }$ (i.e. the experiment duration or 30 steps). $\left\langle N_{f o c}\right\rangle_{\lambda}$ denotes the average number of traction foci with lifetime $\lambda$ for all cells studied in each experimental condition.

\section{Statistics}

Statistical significance was determined using the Kruskall-Wallis test. This test performs a non-parametric one-way ANOVA test to determine if the null hypothesis can be rejected; the null hypothesis states that the distributions from which the two sets of measurements are taken have equal medians. A p-value cutoff of 0.05 was used, below which the null hypothesis was rejected.

\section{RESULTS}

\section{Global traction generation: Effects on force generation at whole-cell level}

Three hydrogel compositions were synthesized to provide cell substrates with different stiffness, further termed low, intermediate, and high stiffness. These corresponded to Young's moduli of 1.4, 2.7, and 4.5 $\mathrm{kPa}$, respectively. The hydrogels were coated with either fibronectin or collagen; there were thus six different experimental conditions.

The total force exerted by each cell at a given time-point was calculated according to Equation 2. Next, these values were averaged over all time-points (i.e. the entire duration of the experiment), providing a single value per cell. Finally, the values for all cells in each condition were averaged. The resulting total forces are shown in Figure 1a. For easier comparison across substrate stiffness, the total force values were normalized to the values obtained at low stiffness (Figure 1b). To confirm that the traction calculation was accurate, the balance of tractions was quantified from the segmented stress footprint for each of the analyzed cells: The magnitude of the vector sum of forces over the stress footprint was compared to the total force. Despite not imposing the biomechanical constraint of zero sum of stresses on the cell with the traction recovery method, there was a minor deviation from zero of the total cell traction of $0.2131 \pm$ $0.2219 \%(\mu \pm \sigma)$ in the X-direction and of $0.1984 \pm 0.2102 \%$ in the Y-direction. Thus the cells find themselves at equilibrium. This suggests that the method of traction recovery is trustworthy. 
Our data reveal an increase in the total force with an increase of substrate stiffness (Figure 1a), in accordance with the literature $(15,30)$. This increase is observed both on fibronectin and collagen. For all stiffness values, average total force was higher on collagen than on fibronectin. This difference was significant at low and high stiffness hydrogels, but not at intermediate stiffness due to larger variability in cell measurements on collagen.

The trend with which force increases with hydrogel stiffness was found to be influenced by the ECM protein used (Figure 1b). On collagen, the increase of average total force is gradual, increasing 1.40 fold at intermediate stiffness and 1.73 fold at high stiffness, relative to total force at low stiffness. With the variability in measurements observed on collagen, these relatively smaller differences compared to those on fibronectin were not detected as significant. In contrast, on fibronectin, a more drastic total force increase was found from low to intermediate stiffness (2.43 fold increase), after which total force barely increased ( 2.51 fold increase with respect to low stiffness). This difference with respect to total force at lowest stiffness, was significant.

Additionally, the maximum traction (Equation 4) was also compared between conditions (Figure S3 in the Supplemental Materials). Unlike total force values, there was no significant difference between fibronectin and collagen for the same stiffness.

a
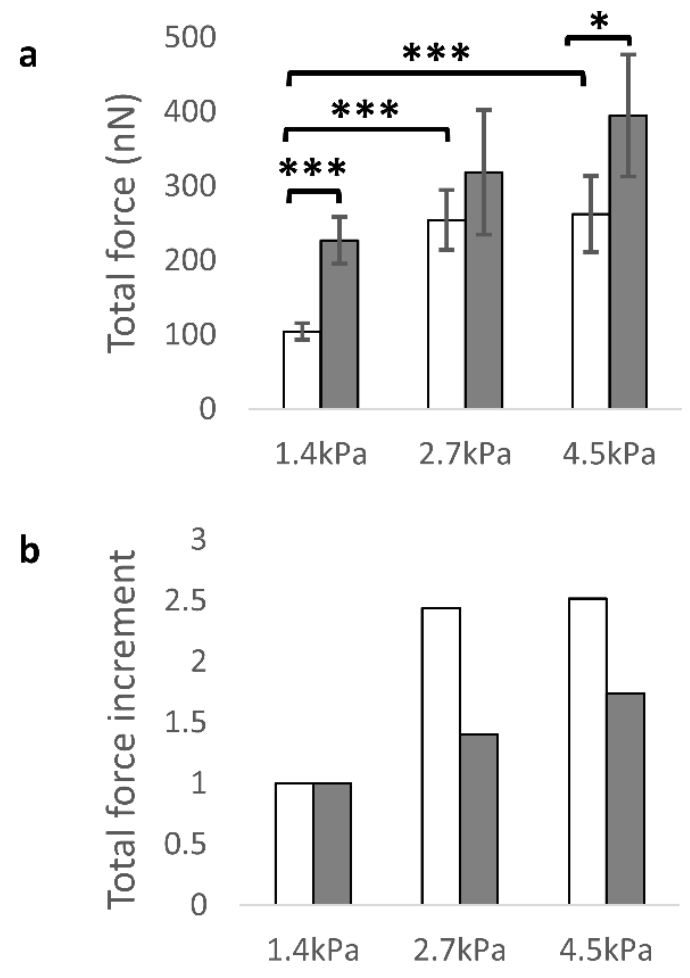

$\square$ FIBRONECTIN $\square$ COLLAGEN

Figure 1. (a) Time-averaged total force. Values for each cell were obtained by averaging the calculated force over all 30 experimental time-steps (2.25 min each). Plots show average (bar) and standard deviation (error bars) of the values calculated for the different cells in each of the experimental conditions. (b) Normalized total force (normalized with respect to total force at low stiffness) of cells on different stiffness hydrogels functionalized with fibronectin or collagen. ( $n=15$ cells for 1.5 and 2.7 $\mathrm{kPa}$ fibronectin, $\mathrm{n}=12$ cells in $4.5 \mathrm{kPa}$ fibronectin and $1.5 \mathrm{kPa}$ collagen, $\mathrm{n}=10$ cells for $2.7 \mathrm{kPa}$ collagen and $\mathrm{n}=11$ cells on $4.5 \mathrm{kPa}$ collagen). Statistical significance: $* p<0.05, * * * p<0.005$ 


\section{Cells display larger force polarization on collagen}

The force dipoles' magnitudes and dipole ratio were calculated for cells in each of the experimental conditions. Average values are presented in Figure 2. The negative sign indicates that the dipole is contractile. The dipole ratio revealed that regardless of hydrogel stiffness, HUVECs on collagen are more polarized than on fibronectin.
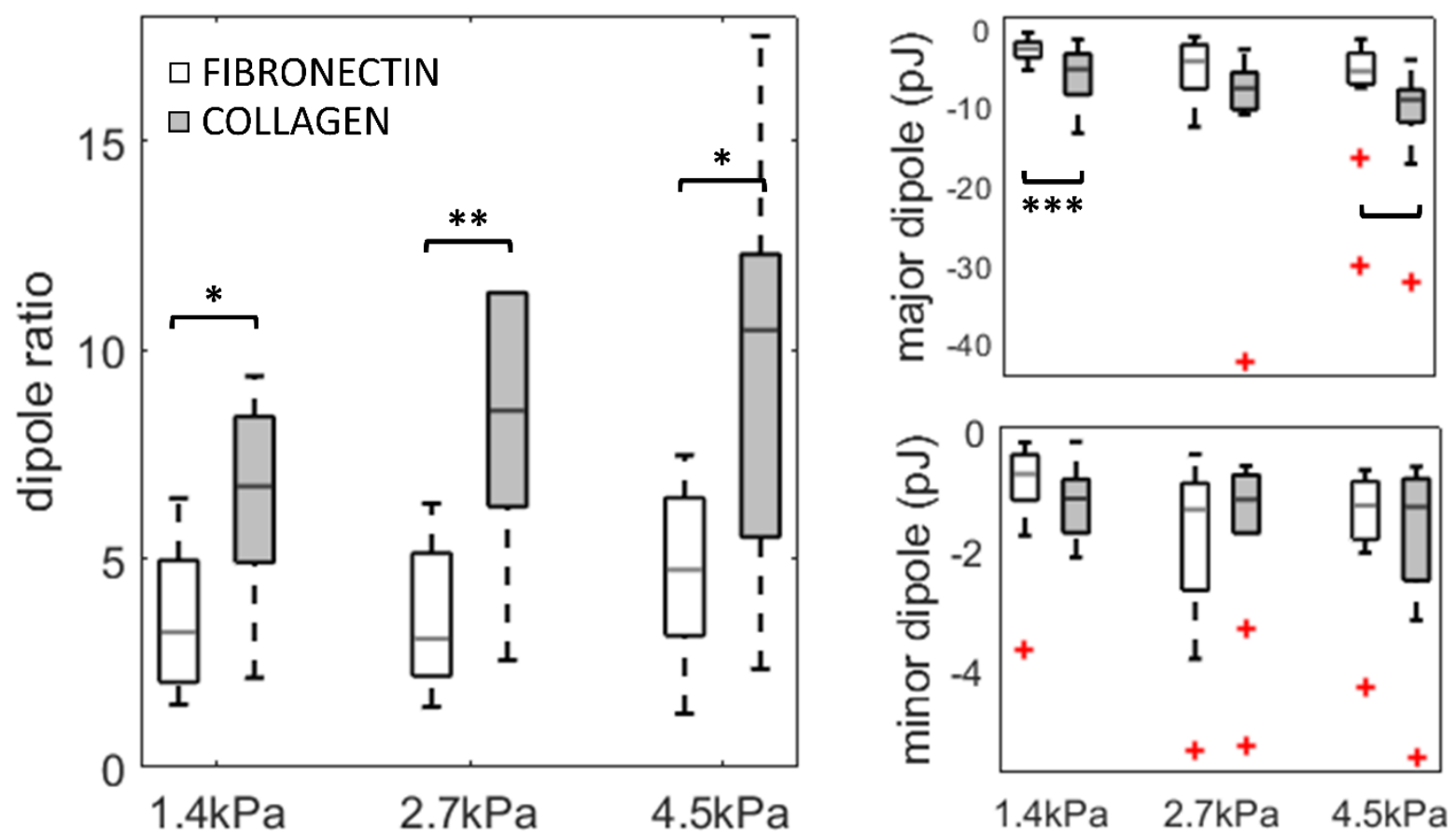

Figure 2. Time-averaged dipole ratio (left) major dipole magnitude (top right), and minor dipole magnitude (bottom right) of cells on different stiffness hydrogels functionalized with fibronectin or collagen. Values for each cell were obtained by averaging dipole values over all 30 experimental time-steps ( $2.25 \mathrm{~min}$ each) and plots show distribution over the different cells in each of the experimental conditions: Central mark indicates median. Top and bottom edges of box indicate first and third quartiles, respectively. Whiskers show extent of lowest and highest values, excluding outliers. Outliers (marked by ' + ' symbol) are not shown for dipole ratio only for displaying purposes. ( $n=15$ cells for 1.5 and $2.7 \mathrm{kPa}$ fibronectin, $n=12$ cells in $4.5 \mathrm{kPa}$ fibronectin and $1.5 \mathrm{kPa}$ collagen, $\mathrm{n}=10$ cells for $2.7 \mathrm{kPa}$ collagen and $\mathrm{n}=11$ cells on $4.5 \mathrm{kPa}$ collagen). Statistical significance: $* p<0.05, * * \mathrm{p}$ $<0.01, * * * p<0.005$

\section{Local traction generation: Effects on force generation at subcellular level}

The molecular adhesion complexes are discrete and can vary in size. Starting with individual integrin molecules binding ECM proteins, these can grow into focal adhesions of a few micrometers in size as they are engaged by the intracellular molecular machinery (31). Given the discrete and subcellular nature of adhesion, we segment the traction footprint into small units we dub traction foci (see Methods section) and evaluate the effect of substrate stiffness and ECM protein on foci behavior. Each traction focus corresponds to part of the traction footprint associated with a single local peak in traction magnitude (Figure 3a).

To determine if the strongest foci behave differently from all other foci, we further made a distinction between the strongest traction focus observed in each cell (from here onwards referred to as strongest 
population) and the entire foci population (from here onwards referred to as whole population). This whole population includes the strongest focus. The strongest focus was defined according to the maximum force exerted by the entire focus at any point during the experiment.

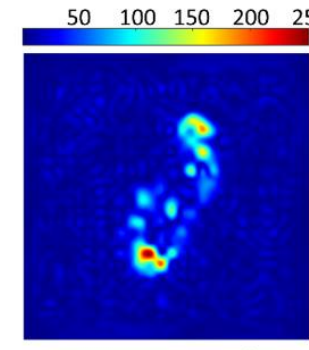

Traction magnitude $\operatorname{map}(\mathrm{Pa})$

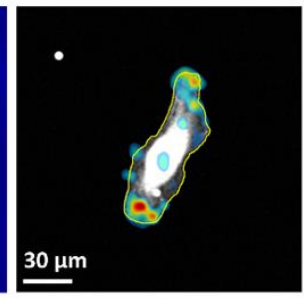

Traction footprint

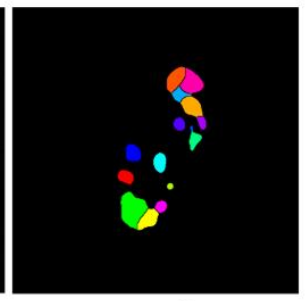

Traction foci
C

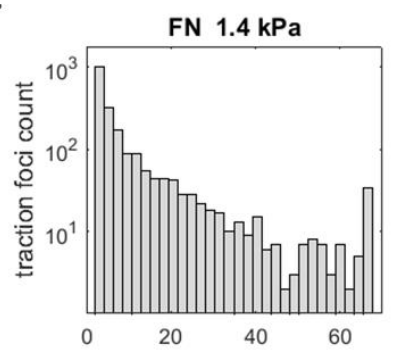

FN $2.7 \mathrm{kPa}$

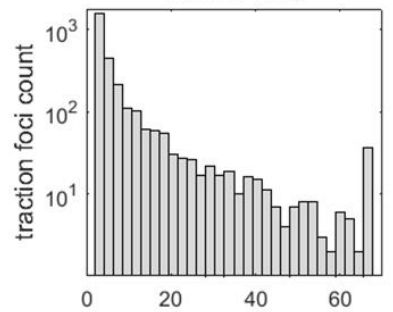

FN $4.5 \mathrm{kPa}$

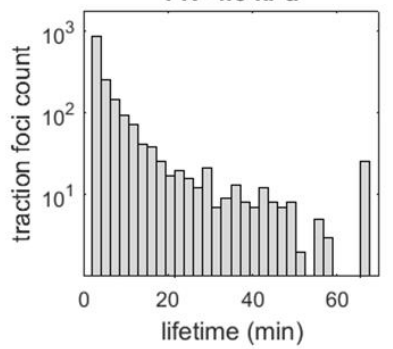

b

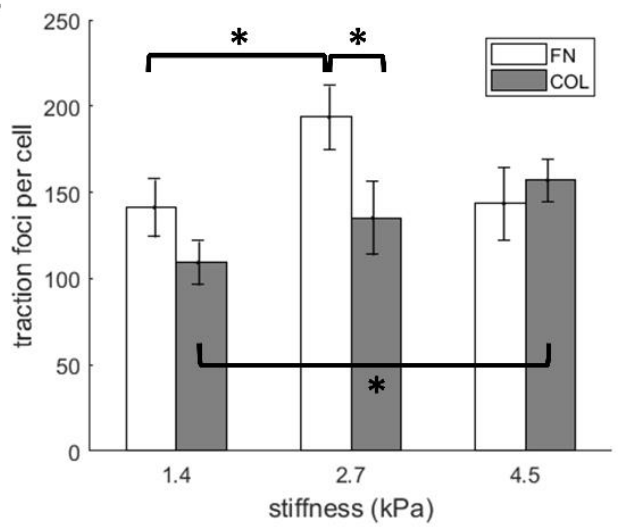

d
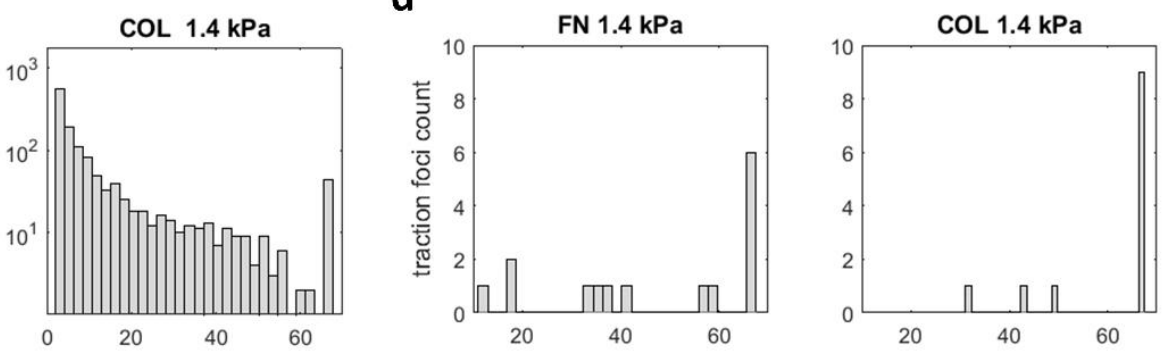

COL $2.7 \mathrm{kPa}$
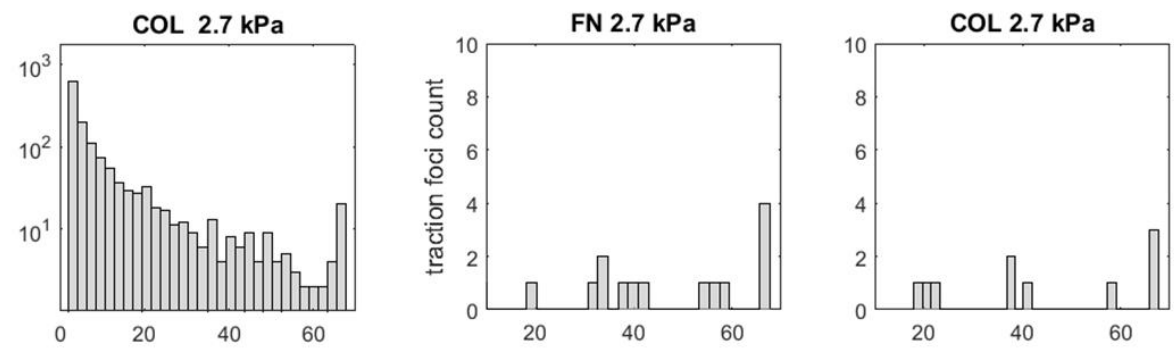

$\mathrm{COL} 4.5 \mathrm{kPa}$
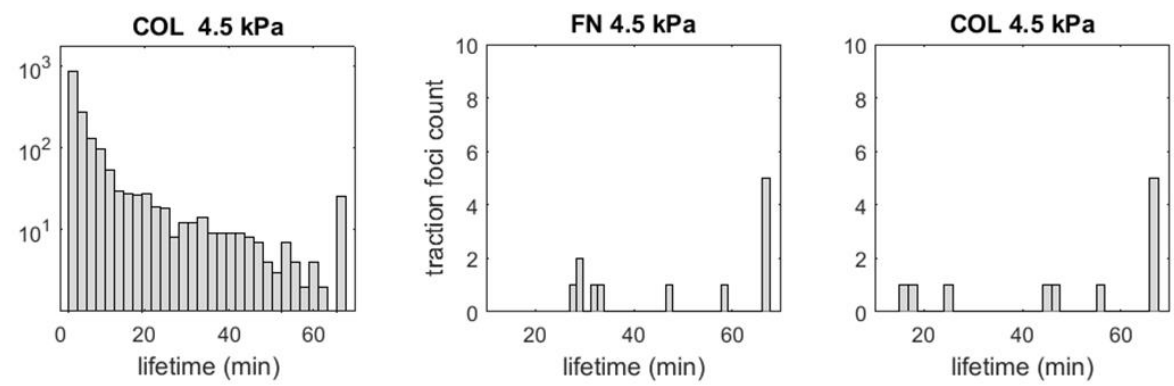

Figure 3. (a) Representative image of the recovered tractions (left), traction footprint overlapped with the cell image (middle), and segmented traction foci (right). (b) Average \pm SD of the total (cumulative) number of traction foci per cell throughout an experiment. (c) Histogram of whole population traction foci as a function of foci lifetime. (d) Histogram of strongest population traction foci as a function of foci lifetime were plotted. (whole population: FN 1.4kPa $n=1098$, COL 1.4kPa $n=757, F N 2.7 \mathrm{kPa}$

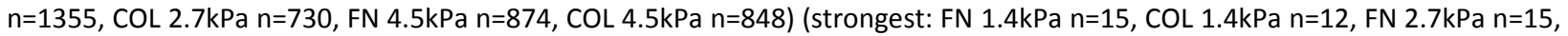
$\operatorname{COL} 2.7 \mathrm{kPa} n=10, \mathrm{FN} 4.5 \mathrm{kPa} n=12, \mathrm{COL} 4.5 \mathrm{kPa} n=11)$. Statistical significance: * $p<0.05$. Abbreviations: collagen (COL), fibronectin (FN). 
First, we quantified the total (i.e. cumulative) number of foci per cell that appeared during the entire duration of the experiment (Figure $3 b$ ). On collagen, this number increased with increasing stiffness to the same extent we saw the total force per cell increase (Figure 1a), but significant when comparing low and high stiffness conditions. In contrast, for cells seeded on fibronectin, this number reached its maximum for the intermediate stiffness $(2.7 \mathrm{kPa})$, a significant increase relative to the low stiffness condition.

Without an external stimulus (e.g. chemoattractant), most of the HUVECs we tracked did not migrate: Approximately $7 \%$ of the cells analyzed migrated a cell-length during the observation period of $68 \mathrm{~min}$ (not shown). This contrasts similar studies performed on more aggressive cells, such as cancer cells (32). The HUVECs did not display clear protrusive front. And although fluctuations with certain periodicity in time were observed in total cell force (not shown), average values over the entire experiment were enough to demonstrate the different behavior observed for the cells in the different experimental conditions: Variations for each cell are smaller than the differences in tractions observed when varying substrate stiffness and coating molecule (with the exception of a few outliers). Ultimately, without a clear migration direction velocity, and finding that temporal averages of traction values over the experimental interval were characteristic for the cell, we decided to quantify temporal dynamics only in terms of lifetime of traction foci. The distribution of the traction foci according to lifetime for the different conditions is presented in Figure 3c. This showed that the additional number of traction foci counted for cells seeded on fibronectin relative to collagen were mostly short-lived foci with lifetimes between 0 and $4.5 \mathrm{~min}$. The strongest foci were not necessarily long-lived (Figure 3d).

\section{Short-lived traction foci contribute significantly to cellular traction}

We also quantified the average force exerted by each of these subpopulations (Figure 4, bars). The mean force of the foci tends to increase with foci lifetime for most experimental conditions. A notable exception was observed in the high stiffness $(4.5 \mathrm{kPa})$ hydrogel coated with fibronectin. In this condition, foci with intermediate lifetimes (between 25-45 min) were the strongest.

Next, the relative contribution of each subpopulation of traction foci to the total force exerted by the cells in each condition was quantified as the percentage total force (Figure 4, solid line); see Equation 8. Even though the short-lived foci constituted the weakest population, they were so abundant that they contributed the most to the total force throughout the experiment (between 22 and 30\%, depending on the condition). 
FN $1.4 \mathrm{kPa}$

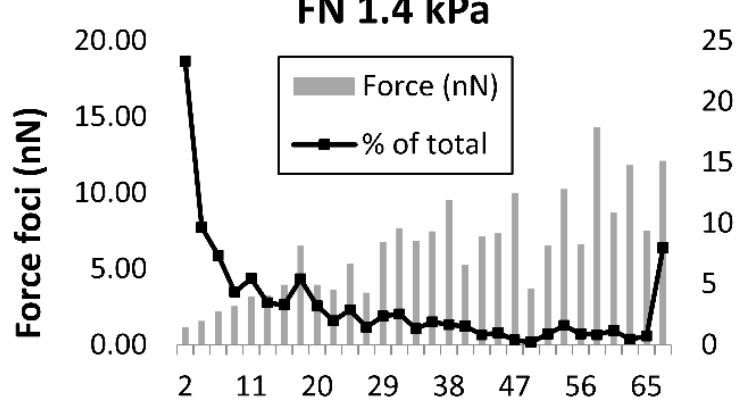

FN $2.7 \mathrm{kPa}$

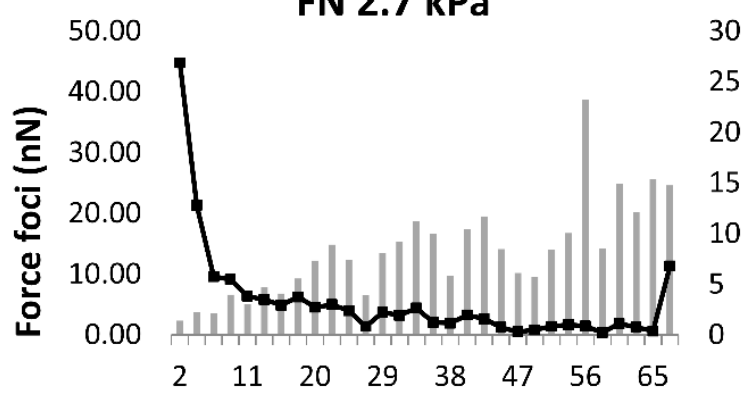

FN 4.5 kPa

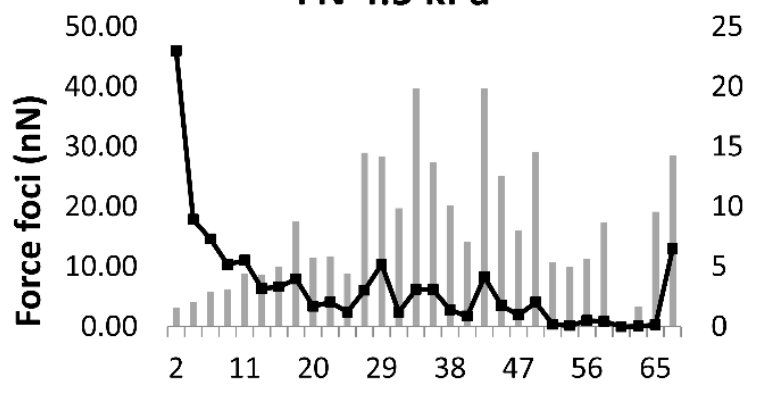

lifetime (min)

\section{COL $1.4 \mathrm{kPa}$}
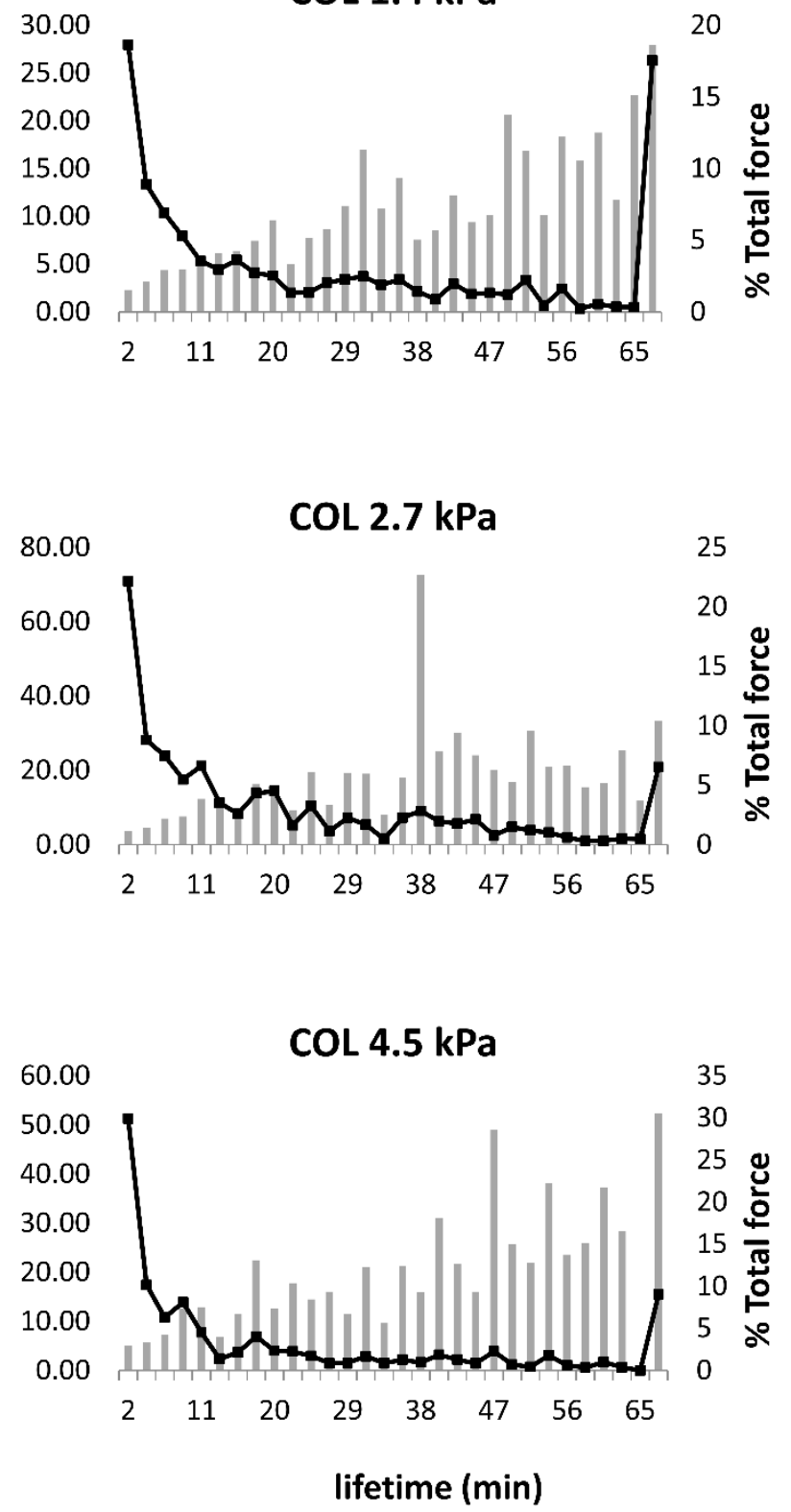

Figure 4. Average force exerted by traction foci with a given lifetime (bars, left vertical axis) and percentage of contribution to the total force exerted by the cell along the whole experiment by each set of traction foci (solid line, right vertical axis) per condition. Abbreviations: collagen (COL), fibronectin (FN).

\section{Effects of hydrogel stiffness on local traction generation}

Figure 5 presents the dependence of area, force, and mean traction of the foci on hydrogel stiffness. No significant difference in foci area was found with varying stiffness (Figures 5a-b). The foci force for both whole and strongest populations ( $5 c-d)$, were found to increase with increasing stiffness on average, although only significantly on fibronectin. Mean focus traction displayed the same trend as foci force with regard to hydrogel stiffness (Figures $5 e-f$ ); this follows from Equation 3. 
a

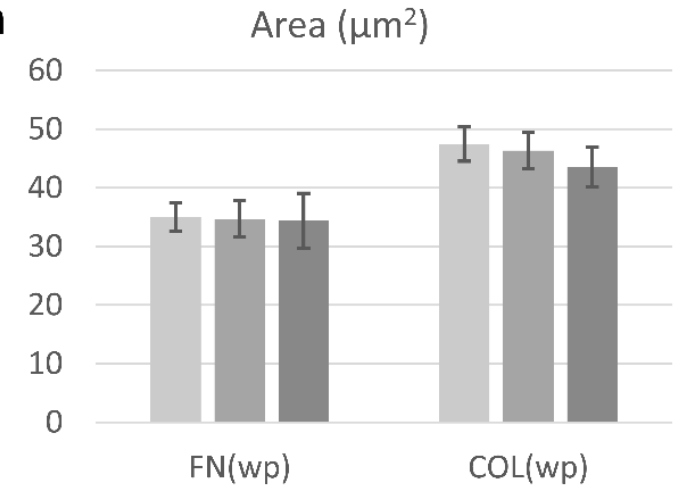

c

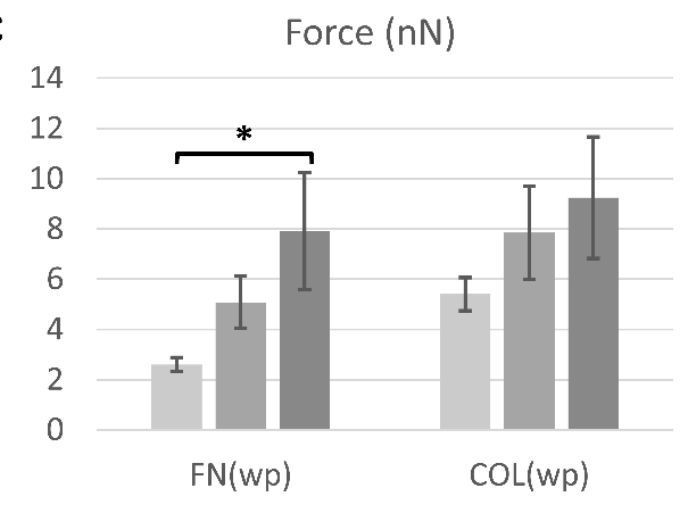

e

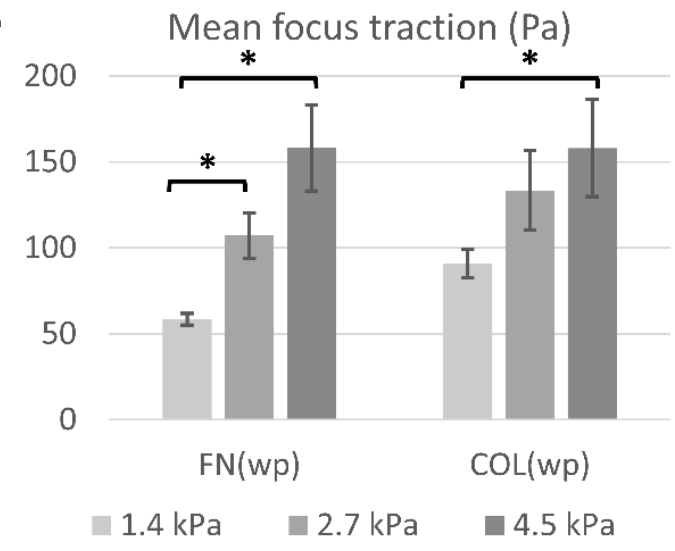

b

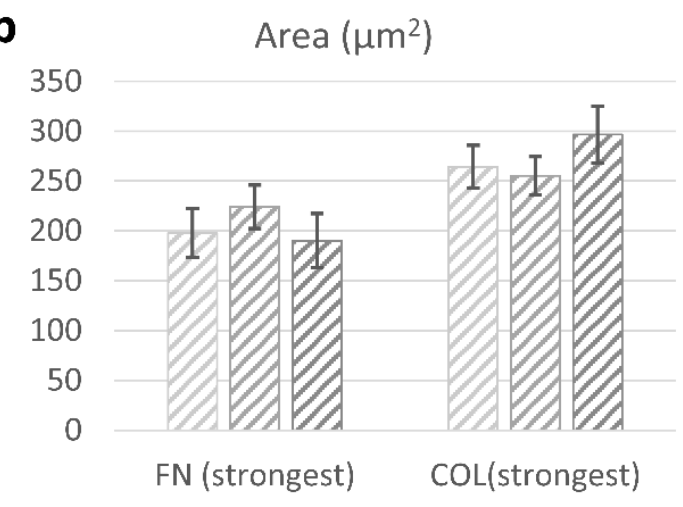

d

d Force $(\mathrm{nN})$

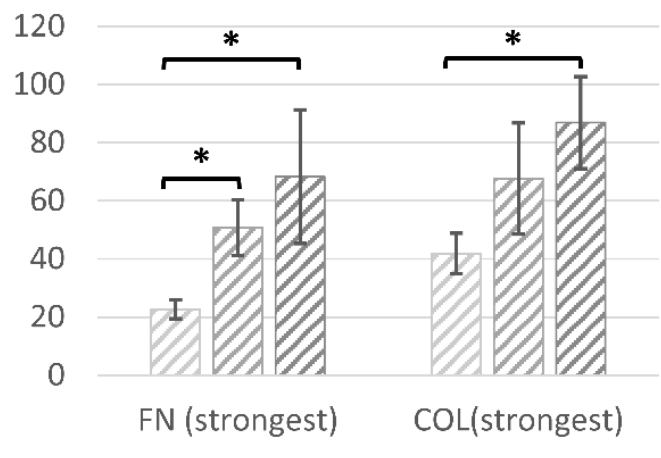

f

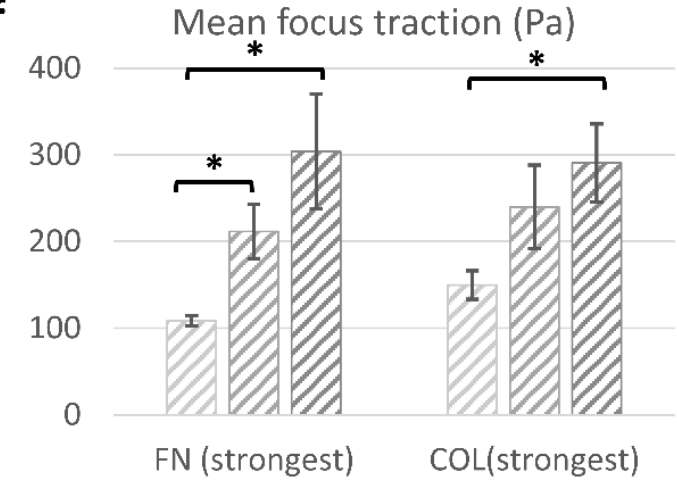

$\square 1.4 \mathrm{kPa} \quad \square 2.7 \mathrm{kPa} \quad \square 4.5 \mathrm{kPa}$

Figure 5. Characterization of traction foci in whole and strongest populations and comparison across the different hydrogel stiffness values: Average area of the traction foci for the whole (a) and (b) strongest populations; Average force exerted by the traction foci for the whole (c) and strongest (d) populations; Mean focus traction for the whole (e) and strongest ( $\mathrm{f}$ ) populations; Average \pm SD were plotted (number of values averaged per condition: $F N 1.4 \mathrm{kPa} n=1098, \mathrm{COL} 1.4 \mathrm{kPa} n=757, \mathrm{FN} \mathrm{2.7kPa} n=1355$, $\mathrm{COL} 2.7 \mathrm{kPa} n=730, \mathrm{FN} 4.5 \mathrm{kPa} n=874, \mathrm{COL} 4.5 \mathrm{kPa} n=848$ ) (strongest: $\mathrm{FN} 1.4 \mathrm{kPa} n=15, \mathrm{COL} 1.4 \mathrm{kPa} n=12, \mathrm{FN} 2.7 \mathrm{kPa} n=15, \mathrm{COL} 2.7 \mathrm{kPa}$ $\mathrm{n}=10, \mathrm{FN} 4.5 \mathrm{kPa} \mathrm{n}=12, \mathrm{COL} 4.5 \mathrm{kPa} \mathrm{n}=11)$. Abbreviations: collagen $(\mathrm{COL})$, fibronectin $(\mathrm{FN})$, whole population (wp). Statistical significance: $* p<0.05$.

\section{Effects of ECM protein on local traction generation}


We also compared area, force, and mean focus traction calculated for the foci between conditions corresponding to different ECM protein but same hydrogel stiffness (Figure 5). The same data was reorganized for ease of comparison and is presented in Figure S5 in the Supplemental Materials.

Traction foci had both a larger area and exert higher forces (although only significantly different for low stiffness) in cells seeded on collagen compared to those on fibronectin (Figures 5a-d and Figure S5a-b). Mean focus traction values were also higher on collagen coated hydrogels on low and intermediate stiffness conditions (again, significantly different for low stiffness), but were equal on the high stiffness condition (Figure $5 e-f$ and Figure S5c).

Comparing the different populations of traction foci reveals that the strongest foci population (i.e. strongest foci per cell in each condition) has an area approximately 6 times larger on average than the whole foci population (Figure 5a-b and Figure S5a). For this reason, we also calculated the mean foci traction as the force of the focus divided by its area (Equation 7); we find that the strongest foci population is exerting 1.9 and 1.8 times (on fibronectin and on collagen, respectively) higher tractions on average compared to the whole foci population, independently of their area. This discards the possibility that larger foci exert higher forces simply because of their larger area.

The relation between area and force for each individual focus is shown in Figure S6 in the Supplemental Materials; in general, we found that there is a linear relationship between foci area and foci force. Time average foci tractions also increase with foci area, but not linearly (Figure S7 in the Supplemental Materials).

\section{DISCUSSION}

Traditionally, cellular tractions are quantified by summing the traction magnitude over the entire footprint, producing a single value per cell. This global analysis reveals HUVECs exert higher total force and maximum traction with increasing hydrogel stiffness (Figure 1). This is consistent with the literature $(15,30,33)$, when observing cells seeded on collagen. The global analysis for cells on fibronectin, displays a different trend (Figure 1b). We see a saturation of the total force response by HUVECs on fibronectin coated hydrogels.

This saturation had been reported for HUVECs by Müller et al. (34). In this study, HUVECs were also grown on hydrogels with three different stiffness values coated with fibronectin: $2.5,5.0$, and $9.0 \mathrm{kPa}$. Total force exerted by the cells was also found to saturate at the intermediate stiffness condition. Unlike in our results, maximum traction was found to also saturate. This led the authors to suggest that a single adhesion site reaches a limit in the force they can exert of approximately $5 \mathrm{kPa}$ and hypothesize that any further increase in global traction exertion could occur through formation of additional "traction force centers." The difference with our cells in the stiffness value at which saturation is observed may arise from methodological differences in the studies, such as cells with a higher passage number and the use of cells that express a fluorescent protein.

Through our local analysis of tractions, we show that the number of traction foci on fibronectin-coated hydrogels increases from low to intermediate stiffness hydrogels, which declines when stiffness was further increased to the high stiffness condition (Figure $3 \mathrm{~b}$ ). At the same time, the average force per traction focus steadily increases with hydrogel stiffness (Figure 4c). Taken together, these observations 
suggest that the decrease in the number of traction foci is balanced by the increase in the average force per focus when going from intermediate to high stiffness, leading to the saturation behavior.

The higher average force per focus on fibronectin-coated high stiffness hydrogels seems to be mostly associated to stronger foci with lifetimes between 25-45 minutes (Figure 4). We confirm that this may be an explanation for the saturation response to increasing stiffness in fibronectin coated hydrogels by multiplying the average number of foci (Figure $3 b$ ) by the average focus force (Figure $5 c$ ): The resulting values are presented in Figure $\mathbf{S} 4$ in the Supplemental Materials. These trends in total force with changing hydrogel stiffness are remarkably similar to those obtained at the whole-cell level (Figure 1).

This saturation in global force was observed in experiments with hydrogels coated with fibronectin, like Müller et al. used, but not with collagen. On fibronectin, the higher number of traction foci observed in the intermediate stiffness condition (in comparison to the low stiffness condition) was accompanied by a higher average force (Figure $5 \mathrm{c}$ ) and traction (Figure 5e). Differences in local response to binding fibronectin and collagen can arise due to multiple sources: Adhesion complex conformation (as different component proteins are recruited into the adhesion complexes as they mature), differential downstream signaling, or the difference between mechanical response of fibronectin and collagen to tension. We speculate that molecular mechanisms underlie these observations, and although these multiple sources may be tied to one another, the initial step is the engagement of different integrin subunits involved in binding to either fibronectin or collagen (35). Members of the integrin family are the initial proteins responsible for binding fibronectin and collagen.

Different integrin dimers are known to bind different ECM molecules (36). Specifically in the case of HUVECs, which bind collagen through the $\alpha_{2} \beta_{1}$ integrin (37); it has been shown that when HUVECs are cultured in the presence of Histidine-Rich Glycoprotein blocks (HRG), which bind the I-domain of the $\alpha 2$ subunit preventing it from binding collagen, adhesion of HUVECs is reduced on collagen by up to $90 \%$. The same does not occur on fibronectin, demonstrating that $\alpha 2 \beta 1$ is the principal integrin dimer responsible for binding of HUVECs on collagen. Meanwhile on fibronectin, in in vitro assays on fibronectin rich cell deposited matrix (in which negligible to minimal expression of collagens I and IV were detected), the combined blocking of $\alpha 5 \beta 1$ and $\alpha v \beta 3$ resulted in almost complete inhibition of vascular morphogenesis, while blocking of $\alpha 5 \beta 1$ alone significantly reduced vascular morphogenesis, and blocking of $\alpha v \beta 3$ alone only slightly retarded vascular morphogenesis (38). This suggests that these integrin dimers are the ones responsible for binding of HUVECs to fibronectin.

Binding of fibronectin by $\alpha_{5} \beta_{1}$ integrin has been shown to occur through a two-step process where the cell adhesion complex goes from a low tension configuration to a high tension configuration, a process dubbed "integrin switch" (39). After initial binding, when large enough tension is applied, the fibronectin molecule extends exposing a synergy site that interacts with the $\alpha_{5}$ subunit. This process leads to the activation of focal adhesion kinase (FAK), which results in the maturation of the FA. Meanwhile, cells bind collagen through the $\alpha_{2} \beta_{1}$ integrin and do not need to exceed a tension threshold for FAK activation (36, 40). More importantly, this integrin switch on fibronectin has been shown to be dependent on substrate stiffness, only occurring on stiffer substrates (39). This difference may provide an explanation for our results: As a consequence of insufficient tension across cell adhesions on our low stiffness hydrogels (1.4 $\mathrm{kPa}$ ) functionalized with fibronectin, a partial activation of FAK leads to a significantly lower number of traction foci (Figure 3b) and a lower average traction per focus (Figure 5e) than that calculated in intermediate stiffness hydrogels. On the intermediate stiffness hydrogels, the tension the fibronectin 
molecule is experiencing could be large enough to fully activate FAK, potentially allowing the cells to exert higher tractions. While such mechanism could partially explain the differences observed between fibronectin-coated low and intermediate stiffness hydrogels, it does not exclude the contribution from other mechanosensitive molecules like talin to FA maturation and traction buildup (41), that act regardless of hydrogel coating (42) and are responsible for the common increase in traction with increasing stiffness. Further studies focusing on inhibition of integrins or FAK signaling may provide insight as to whether our results can be attributed to this particular molecular mechanism.

By choosing the term traction focus, we purposely avoid making a conjecture about the biological identity of the traction sources in our HUVECs. Because we coat the entire surface of the hydrogel with ECM proteins (Figure S1), there is no limitation to the size of FA clustering that may occur nor a minimal distance that must exist between adhesions. This allowed us to characterize the range in the area of the cell-substrate interface over which a HUVEC can exert peak tractions as well as the range in force locally, but it limited the amount of comparisons we can perform with studies in the literature. Immunostaining for fibronectin and collagen reveal that despite coating entirely the hydrogel surface, the distribution of the proteins differs. While the distribution of fibronectin is homogeneous, that of collagen is heterogeneous with larger groupings of the protein present over the more prevalent uniform distribution (Figure S1). This is not surprising given the thicker fibrils formed by collagen compared to fibronectin. The larger of these collagen groupings are comparable in size to the larger traction foci; we do not discount that this may also play a role in determining traction foci size.

The work by Fu et al. used a micropost array ( $2 \mu \mathrm{m}$ in diameter) to characterize the size of single FA complexes as well as the traction exerted by each complex in HUVECs (43). In this study, the authors measure the size of FAs that have an area of up to $2.50{\mu \mathrm{m}^{2}}^{2}$ and tractions reaching values up to $10 \mathrm{nN} / \mu \mathrm{m}^{2}$. In our experiments traction foci were found to have areas ranging between 0.2-665 $\mu \mathrm{m}^{2}$ and exert forces ranging between $0.14-95 \mathrm{nN}$. This suggested that individual traction foci could correspond to a cluster of multiple FAs, which we later confirmed and will discuss later. The force values per foci seem comparable to those obtained by Fu et al.; as few as 10 of the strongest FA observed by Fu et al. could account for the force of the strongest foci observed in our study. In our results we found there was no difference in area of individual traction foci with stiffness (Figure 5a), but there was an increase in force (Figure 5c). This indicates that increased force arises from maturation of FAs (i.e. recruitment of additional proteins and engagement of the cytoskeleton), possibly accompanied by clustering of multiple FAs. We found that traction foci have lifetimes in the same order of magnitude as FAs. The mean foci lifetime was around 10 minutes, and between 40-60 minutes in the case of the strongest foci (Figure S5). For example, in the case of fibroblasts, nascent adhesions form for a period between 1-2 min, and if they mature they can lifetimes ranging from $20-40 \mathrm{~min}(44,45)$.

Other studies have used experimental conditions closer to ours but quantified FA size and traction for other cell types. A study by Hernández-Varas et al. measured the area of individual FAs for H1299 (nonsmall lung carcinoma) cells on fibronectin coated glass as well as the tension using a vinculin Förster Resonance Energy Transfer (FRET) molecular tension probe (20); in it, without a limit on FA area, cells were found to have FA areas ranging between 0.20-6 $\mu \mathrm{m}^{2}$. This suggests that even in the absence of a physical constraint to FA size, our largest traction foci could correspond to a cluster of FAs. Unfortunately, the authors report tension in the FAs in arbitrary units, thus preventing us from making a comparison with our traction values. Another study, by Stricker et al., seeded U2OS (human osteosarcoma) cells and NIH 3T3 (murine embryonic) fibroblasts on fibronectin-coated PA hydrogels of 2.8 and $16 \mathrm{kPa}$, respectively 
(26). This study deserves a special mention because amongst the methods it implements for traction force calculation is Traction Reconstruction with Point Force (TRPF), introduced originally by Schwarz et al. (46). This method calculates tractions from recovered displacements by constraining the local stresses to the area of segmented focal adhesions, thus also analyzing cellular tractions at the subcellular level while using traditional TFM. Similar to Hernández-Varas et al., the authors found distinct phases of FA growth and correlation with traction build-up. Individual FAs (comparable in size to those characterized by Fu et al.) had traction values reaching $500 \mathrm{~Pa}$. These values are larger than those we report. This could be due to the cell type, as fibroblasts can exert high tractions. The highest mean focus traction we observed was close to $300 \mathrm{~Pa}$ in $4.5 \mathrm{kPa}$ gels, regardless of ECM protein (Figure $5 \mathrm{f}$ ). Although this method reduces the need for regularization schemes used to avoid overfitting of the recovered force field to the errors present in the calculated displacements, its reliability highly depends on capturing all the focal adhesions involved in the force-related cell-matrix interactions and on the accuracy of their subsequent segmentation (16). This is challenging and may require additional equipment, such as additional lasers (i.e. different wavelengths) to reliably detect fiducial markers in the substrate as well as focal adhesions. With our method, in comparison to TRPF, we seek to avoid these technical challenges.

For a direct comparison between focal adhesions and traction foci, we imaged focal adhesions through a fluorescent vinculin reporter. Video S2, in the Supplemental Materials, presents a HUVEC cultured on a $2.7 \mathrm{kPa}$ hydrogel coated with collagen in which the fluorescent focal adhesions are enhanced and shown along with the outline of the traction foci. This video demonstrates that each traction focus corresponds to the traction distribution exerted by at least one, but in most cases multiple focal adhesions, often at the edges of the foci. Video S3, in the Supplemental Materials, shows the segmented focal adhesions overlaid on the stress footprint of the cell. This video shows how the strongest foci are associated with the largest focal adhesions. It is not possible to determine if these large adhesions are really multiple clustered adhesions or individual ones that have matured and simultaneously grown. Observing the evolution of these focal adhesions in time, shows that they break up into smaller ones, suggesting that they are clusters of focal adhesions.

In addition to providing insight on the total force measurements at the global scale, looking at traction foci can also help interpret findings on cellular traction orientation through the force dipole. Our results suggest that the ECM protein can affect polarization of cellular forces (Figure 2a). Considering that cells exert higher total forces on collagen (Figure 1a), we saw that increased cellular forces occur simultaneously with increased force polarization (Figure 2). The spatial distribution of cellular tractions has often been presented as synonymous with cellular morphology because it has been thoroughly shown that cells adopting a polarized shape can exert larger tractions. These studies were performed using experimental systems that constrain cell shape (47-49) or expose cells to an external directional stimulus, such as a chemical or a substrate stiffness gradient $(33,50)$. Our experimental system does not attempt to do this, placing cells in a homogeneous environment.

We found that differences in polarization between cells cultured on the two different ECM proteins are obscured if polarization is described solely in terms of morphology. The aspect ratio (i.e. the ratio of major axis to minor axis) was calculated as a measure of shape polarization. Figure S8 in the Supplemental Materials, shows the average aspect ratio of cells in the different experimental conditions. No significant difference in aspect ratio with ECM protein was found for cells seeded in the low and intermediate stiffness conditions. Figure $\mathrm{S} 8$ shows a sample dipole ratio tracked in time along with the aspect ratio: These two quantities were similar but not identical for HUVECs. 
In conclusion, we have demonstrated that more refined spatiotemporal analyses of traction foci at a subcellular level can help in identifying effects of substrate stiffness and coating on traction exertion, as well as in explaining observed differences in cellular forces at a global cell level. While we were successful in explaining the trend with which whole-cell forces varied with stiffness for fibronectin versus collagen, the molecular mechanisms behind these trends remain unknown. Future work should clarify this mechanisms, possibly performing TFM while blocking specific integrin dimers. Imaging of the actin cytoskeleton would also be insightful because acto-myosin contraction along stress fibers is the source of the largest tractions. Even without molecular imaging, however, our results open the door to further analyses at this local level using TFM.

\section{ACKNOWLEDGMENTS}

AIA, DAV, and HVO designed research; AIA, DAV, RS, and MV performed research; DAV and AJP developed analytic tools; AIA and DAV analyzed data; AIA, DAV, AJP, and HVO wrote the manuscript. Ben De Coninck was involved in carrying out additional experiments with the vinculin reporter.

All authors declare that there are no known conflicts of interest associated with this submission. Funding for this work comes from the European Research Council (FP7/2007-2013)/ ERC Grant Agreement $\left(n^{\circ}\right.$ 308223), FWO-Vlaanderen (grants $n^{\circ}$ G.0821.13, G.087018N, and G.0B96.15), KU Leuven internal funding (IDO/13/016), and FWO and EU's Horizon 2020 research and innovation programme (Marie SkłodowskaCurie Grant Agreement $n^{\circ}$ 665501).

\section{REFERENCES}

1. Nishida, N., H. Yano, T. Nishida, T. Kamura, and M. Kojiro. 2006. Angiogenesis in cancer. Vasc. Health Risk Manag. 2: 213-219.

2. Capitão, M., and R. Soares. 2016. Angiogenesis and Inflammation Crosstalk in Diabetic Retinopathy. J. Cell. Biochem. 117: 2443-2453.

3. Carmeliet, P. 2003. Angiogenesis in health and disease. Nat. Med. 9: 653-60.

4. Saharinen, P., L. Eklund, K. Pulkki, P. Bono, and K. Alitalo. 2011. VEGF and angiopoietin signaling in tumor angiogenesis and metastasis. Trends Mol. Med. 17: 347-362.

5. Cross, M.J., and L. Claesson-Welsh. 2001. FGF and VEGF function in angiogenesis: Signalling pathways, biological responses and therapeutic inhibition. Trends Pharmacol. Sci. 22: 201-207.

6. Hirota, K., and G.L. Semenza. 2006. Regulation of angiogenesis by hypoxia-inducible factor 1. Crit. Rev. Oncol. Hematol. 59: 15-26.

7. De Bock, K., M. Georgiadou, and P. Carmeliet. 2013. Role of endothelial cell metabolism in vessel sprouting. Cell Metab. 18: 634-647.

8. Krishnan, L., C.J. Underwood, S. Maas, B.J. Ellis, T.C. Kode, J.B. Hoying, and J.A. Weiss. 2008. Effect of mechanical boundary conditions on orientation of angiogenic microvessels. Cardiovasc. Res. 78: 324-332.

9. Galie, P.A., D.-H.T. Nguyen, C.K. Choi, D.M. Cohen, P.A. Janmey, and C.S. Chen. 2014. Fluid shear stress threshold regulates angiogenic sprouting. Proc. Natl. Acad. Sci. 111: 7968-7973.

10. Mason, B.N., A. Starchenko, R.M. Williams, L.J. Bonassar, and C.A. Reinhart-King. 2013. Tuning 
three-dimensional collagen matrix stiffness independently of collagen concentration modulates endothelial cell behavior. Acta Biomater. 9: 4635-4644.

11. Wu, Y., M.A. Al-Ameen, and G. Ghosh. 2014. Integrated Effects of Matrix Mechanics and Vascular Endothelial Growth Factor (VEGF) on Capillary Sprouting. Ann. Biomed. Eng. 42: 1024-1036.

12. Reinhart-King, C.A., M. Dembo, and D.A. Hammer. 2003. Endothelial Cell Traction Forces on RGDDerivatized Polyarylamide Substrata. Langmuir. 19: 1573-1579.

13. Shiu, Y.-T., S. Li, W.A. Marganski, S. Usami, M.A. Schwartz, Y.-L. Wang, M. Dembo, and S. Chien. 2004. Rho Mediates the Shear-Enhancement of Endothelial Cell Migration and Traction Force Generation. Biophys. J. 86: 2558-2565.

14. Reinhart-King, C.A., M. Dembo, and D.A. Hammer. 2005. The Dynamics and Mechanics of Endothelial Cell Spreading. Biophys. J. 89: 676-689.

15. Califano, J.P., and C.A. Reinhart-King. 2010. Substrate stiffness and cell area predict cellular traction stresses in single cells and cells in contact. Cell. Mol. Bioeng. 3: 68-75.

16. Sabass, B., M.L. Gardel, C.M. Waterman, and U.S. Schwarz. 2008. High resolution traction force microscopy based on experimental and computational advances. Biophys. J. 94: 207-220.

17. Legant, W.R., C.K. Choi, J.S. Miller, L. Shao, L. Gao, E. Betzig, and C.S. Chen. 2013. Multidimensional traction force microscopy reveals out-of-plane rotational moments about focal adhesions. Proc. Natl. Acad. Sci. 110: 881-886.

18. Han, S.J., Y. Oak, A. Groisman, and G. Danuser. 2015. Traction microscopy to identify force modulation in subresolution adhesions. Nat. Methods. 12: 653-656.

19. Plotnikov, S.V., A.M. Pasapera, B. Sabass, and C.M. Waterman. 2012. Force Fluctuations within Focal Adhesions Mediate ECM-Rigidity Sensing to Guide Directed Cell Migration. Cell. 151: 15131527.

20. Hernández-Varas, P., U. Berge, J.G. Lock, and S. Strömblad. 2015. A plastic relationship between vinculin-mediated tension and adhesion complex area defines adhesion size and lifetime. Nat. Commun. 6: 7524.

21. Bergert, M., T. Lendenmann, M. Zündel, A.E. Ehret, D. Panozzo, P. Richner, D.K. Kim, S.J.P. Kress, D.J. Norris, O. Sorkine-Hornung, E. Mazza, D. Poulikakos, and A. Ferrari. 2016. Confocal reference free traction force microscopy. Nat. Commun. 7: 12814.

22. Tse, J.R., and A.J. Engler. 2010. Preparation of hydrogel substrates with tunable mechanical properties. Curr. Protoc. Cell Biol. 47: 1-16.

23. Garcia, D. 2010. Robust smoothing of gridded data in one and higher dimensions with missing values. Comput Stat Data Anal. 54: 1167-1178.

24. Butler, J.P., I.M. Tolić-Nørrelykke, B. Fabry, and J.J. Fredberg. 2002. Traction fields, moments, and strain energy that cells exert on their surroundings. Am. J. Physiol. Cell Physiol. 282: C595-605.

25. Schwarz, U.S., and J.R.D. Soiné. 2015. Traction force microscopy on soft elastic substrates: A guide to recent computational advances. Biochim. Biophys. Acta - Mol. Cell Res. 1853: 30953104. 
26. Stricker, J., Y. Aratyn-Schaus, P.W. Oakes, and M.L. Gardel. 2011. Spatiotemporal Constraints on the Force-Dependent Growth of Focal Adhesions. Biophys. J. 100: 2883-2893.

27. Dembo, M., and Y.-L. Wang. 1999. Stresses at the Cell-to-Substrate Interface during Locomotion of Fibroblasts. Biophys. J. 76: 2307-2316.

28. Ambrosi, D. 2006. Cellular Traction as an Inverse Problem. SIAM J. Appl. Math. 66: 2049-2060.

29. Tanimoto, H., and M. Sano. 2014. A Simple Force-Motion Relation for Migrating Cells Revealed by Multipole Analysis of Traction Stress. Biophys. J. 106: 16-25.

30. Ghibaudo, M., A. Saez, L. Trichet, A. Xayaphoummine, J. Browaeys, P. Silberzan, A. Buguin, and B. Ladoux. 2008. Traction forces and rigidity sensing regulate cell functions. Soft Matter. 4: 1836.

31. Choi, C.K., M. Vicente-Manzanares, J. Zareno, L.A. Whitmore, A. Mogilner, and A.R. Horwitz. 2008. Actin and alpha-actinin orchestrate the assembly and maturation of nascent adhesions in a myosin II motor-independent manner. Nat. Cell Biol. 10: 1039-1050.

32. Peschetola, V., V.M. Laurent, A. Duperray, R. Michel, D. Ambrosi, L. Preziosi, and C. Verdier. 2013. Time-dependent traction force microscopy for cancer cells as a measure of invasiveness. Cytoskeleton. 70: 201-214.

33. Lo, C.-M., H.-B. Wang, M. Dembo, and Y. Wang. 2000. Cell Movement Is Guided by the Rigidity of the Substrate. Biophys. J. 79: 144-152.

34. Müller, C., and T. Pompe. 2015. Distinct impacts of substrate elasticity and ligand affinity on traction force evolution. Soft Matter. 12: 272-80.

35. Luo, B.-H., C. V Carman, and T.A. Springer. 2007. Structural basis of integrin regulation and signaling. Annu. Rev. Immunol. 25: 619-47.

36. Seong, J., A. Tajik, J. Sun, J.-L. Guan, M.J. Humphries, S.E. Craig, A. Shekaran, A.J. García, S. Lu, M.Z. Lin, N. Wang, and Y. Wang. 2013. Distinct biophysical mechanisms of focal adhesion kinase mechanoactivation by different extracellular matrix proteins. Proc. Natl. Acad. Sci. U. S. A. 110: 19372-7.

37. Roche, F., K. Sipilä, S. Honjo, S. Johansson, S. Tugues, J. Heino, and L. Claesson-Welsh. 2015. Histidine-rich glycoprotein blocks collagen-binding integrins and adhesion of endothelial cells through low-affinity interaction with $\alpha 2$ integrin. Matrix Biol. 48: 89-99.

38. Hielscher, A., K. Ellis, C. Qiu, J. Porterfield, and S. Gerecht. 2016. Fibronectin Deposition Participates in Extracellular Matrix Assembly and Vascular Morphogenesis. PLoS One. 11: e0147600.

39. Friedland, J.C., M.H. Lee, and D. Boettiger. 2009. Mechanically Activated Integrin Switch Controls $\alpha 5 \beta 1$ Function. Science (80-. ). 323: 642-644.

40. Indra, I., and K.A. Beningo. 2011. An in vitro correlation of metastatic capacity, substrate rigidity, and ECM composition. J. Cell. Biochem. 112: 3151-3158.

41. Elosegui-Artola, A., R. Oria, Y. Chen, A. Kosmalska, C. Pérez-González, N. Castro, C. Zhu, X. Trepat, and $P$. Roca-Cusachs. 2016. Mechanical regulation of a molecular clutch defines force transmission and transduction in response to matrix rigidity. Nat. Cell Biol. 18: 540-548. 
42. Kotecki, M., A.S. Zeiger, K.J. Van Vliet, and I.M. Herman. 2010. Calpain- and talin-dependent control of microvascular pericyte contractility and cellular stiffness. Microvasc. Res. 80: 339-348.

43. Fu, J., Y. Wang, M.T. Yang, R.A. Desai, X. Yu, Z. Liu, and C.S. Chen. 2010. Mechanical regulation of cell function with geometrically modulated elastomeric substrates. Nat. Methods. 7: 733-736.

44. Kaverina, I., O. Krylyshkina, and J. V Small. 1999. Microtubule targeting of substrate contacts promotes their relaxation and dissociation. J. Cell Biol. 146: 1033-44.

45. Doyle, A.D., N. Carvajal, A. Jin, K. Matsumoto, and K.M. Yamada. 2015. Local 3D matrix microenvironment regulates cell migration through spatiotemporal dynamics of contractilitydependent adhesions. Nat. Commun. 6: 8720.

46. Schwarz, U.S., N.Q. Balaban, D. Riveline, A. Bershadsky, B. Geiger, and S.A. Safran. 2002. Calculation of Forces at Focal Adhesions from Elastic Substrate Data: The Effect of Localized Force and the Need for Regularization. Biophys. J. 83: 1380-1394.

47. Rape, A.D., W. Guo, and Y. Wang. 2011. The regulation of traction force in relation to cell shape and focal adhesions. Biomaterials. 32: 2043-2051.

48. Oakes, P.W., S. Banerjee, M.C. Marchetti, and M.L. Gardel. 2014. Geometry Regulates Traction Stresses in Adherent Cells. Biophys. J. 107: 825-833.

49. Han, S.J., K.S. Bielawski, L.H. Ting, M.L. Rodriguez, and N.J. Sniadecki. 2012. Decoupling Substrate Stiffness, Spread Area, and Micropost Density: A Close Spatial Relationship between Traction Forces and Focal Adhesions. Biophys. J. 103: 640-648.

50. Jannat, R.A., G.P. Robbins, B.G. Ricart, M. Dembo, and D.A. Hammer. 2010. Neutrophil adhesion and chemotaxis depend on substrate mechanics. J. Phys. Condens. Matter. 22: 194117. 


\section{Spatiotemporal analyses of cellular tractions describe subcellular effect of substrate stiffness and coating}

Alicia Izquierdo-Álvarez ${ }^{1 *}$, Diego A. Vargas ${ }^{1 *}$, Álvaro Jorge-Peñas ${ }^{1}$, Ramesh Subramani $^{2}$, Marie-Mo Vaeyens ${ }^{1}$, and Hans Van Oosterwyck ${ }^{1,3+}$

1. Biomechanics Section, KU Leuven, Celestijnenlaan 300C, 3001 Leuven, Belgium

2. MeBioS, KU Leuven, Kasteelpark Arenberg 30, 3001 Leuven, Belgium

3. Prometheus, Division of Skeletal Tissue Engineering, KU Leuven, Herestraat 49, 3000 Leuven, Belgium

* Alicia Izquierdo-Álvarez and Diego A. Vargas have contributed equally to this work.

† Corresponding author

SUPPLEMENTAL MATERIALS

\section{Supplemental Text}

\section{Hydrogel preparation}

Polyacrylamide (PA) hydrogels were prepared as described by Tse et al. (1): The glass bottom of petri dishes were treated with 3-aminopropyltrimethoxysilane (APTS, Sigma-Aldrich, St. Louis, MO) and 1\% glutaraldehyde. To obtain the desired stiffness values (1.4, 2.7, and $4.5 \mathrm{kPa}$ ), hydrogels were prepared by mixing different volumes of $40 \%$ acrylamide, $2 \%$ bis-acrylamide (Bio-Rad, Hercules, California) with distilled water; volumes are shown in Error! Reference source not found.. After sonication of the mixture, $10 \%$ ammonium persulfate (Bio-Rad, Hercules, California) and TEMED (Sigma-Aldrich, St. Louis, MO) were added to the Bis/Acrylamide mix. Each gel had a volume of $150 \mu \mathrm{L}$; the thickness of the gel (i.e. height from contact with the glass to the protein coating) varied between 100-150 $\mu \mathrm{m}$. The area of the coated surface to which cells bind is approximately $1 \mathrm{~cm}^{2}$.

Table S1: Mixtures of Acrylamide and Bis-Acrylamide (final concentrations) to obtain hydrogels with the corresponding Young's Modulus, as measured by atomic force microscopy (average \pm SD).

\begin{tabular}{|c|c|c|}
\hline $\begin{array}{c}\text { Young's } \\
\text { Modulus (kPa) }\end{array}$ & $\begin{array}{c}\text { Acrylamide } \\
\text { (wt \%) }\end{array}$ & $\begin{array}{c}\text { Bis-Acrylamide } \\
\text { (wt \%) }\end{array}$ \\
\hline $1.4 \pm 0.1$ & 5 & 0.1 \\
\hline $2.7 \pm 0.2$ & 4 & 0.3 \\
\hline $4.5 \pm 0.4$ & 5 & 0.15 \\
\hline
\end{tabular}

These stiffness values were chosen due to a technical limitation of our current methods: Below $1.4 \mathrm{kPa}$, hydrogels (purposely made thin for cell imaging) were brittle and cracked. Above $4.5 \mathrm{kPa}$, cells only caused 
negligible displacements. Human umbilical vein endothelial cells (HUVECs) do not exert as much force as other commonly used cells, e.g. fibroblasts.

As fiducial markers for TFM, $200 \mathrm{~nm}$ carboxylate-modified microspheres (FluoSpheres ${ }^{\circledR}$, ThermoFisher Scientific, Waltham, MA) were added to the mixture at a 1:60 ratio and $15 \mu$ lof the mixture was placed on top of the glass. A Sigmacote ${ }^{\circledR}$-pretreated (Sigma-Aldrich, St. Louis, MO) $12 \mathrm{~mm}$ coverslip was used to flatten the hydrogels. After $30 \mathrm{~min}$ of polymerization, some distilled water was added, and the coverslips were gently removed.

\section{Hydrogel functionalization}

Hydrogels were functionalized with either fibronectin or collagen: First, a dilution of $0.5 \mathrm{mg} / \mathrm{ml}$ of sulphoSANPAH (ThermoFisher Scientific, Waltham, MA) was used. Sulpho-SANPAH was activated with a 3UV light lamp (115 V, $60 \mathrm{~Hz}, 365 \mathrm{~nm}$ wavelength; ThermoFisher Scientific) for $20 \mathrm{~min}$. This was done twice. Next, the hydrogels were washed with sterile PBS before adding the ECM protein such that it saturated the hydrogel surface: Human fiibronectin (Sigma-Aldrich, St. Louis, MO) was added at a concentration of $5 \mu \mathrm{g} / \mathrm{ml}$, concentration above which no difference in distribution of protein was observed (not shown). Rat tail type I collagen (BD bioscience, San Jose, CA) was added at a concentration of $100 \mu \mathrm{g} / \mathrm{ml}$; this concentration was chosen because it is a widely used value in the literature including in work with HUVECs (2-5). Immunostaining of the ECM proteins confirm saturation of the hydrogel surface (Figure S1, Supplemental Materials).

\section{Protein coating immunostaining}

To visualize the protein coating in order to confirm saturation of the cell-substrate interface with the ECM protein, immunostaining was performed on coated hydrogels without cells. Primary antibody to fibronectin (F3648) was purchased from Merck (Kenilworth, NJ, USA). Primary incubation was performed overnight at $4^{\circ} \mathrm{C}$ on an orbital shaker; dilution used was 1:400 in PBST (0.1\% Tween 20,1\% FBS). Primary antibody to collagen (ab34710) was purchased from Abcam (Cambridge, MA, USA). Primary incubation was performed overnight at $4^{\circ} \mathrm{C}$ on a shaker; dilution used was $1: 500$ in PBST $(0.1 \%$ Tween $20,1 \% \mathrm{FBS})$. The same secondary goat anti-rabbit IgG, conjugated with AlexaFluor ${ }^{\circledR} 594$, was used in both cases (A11037), purchased from ThermoFisher Scientific (Waltham, MA, USA). Incubation was performed for $1 \mathrm{~h}$ at room temperature on an orbital shaker.

\section{Atomic Force Microscopy}

The hydrogel stiffness was determined by means of Atomic Force Microscopy (AFM). A JPK Nanowizard ${ }^{\circledR}$ III Bioscope-AFM (JPK Instruments AG, Berlin, Germany) was employed to obtain the Young's modulus of the PA hydrogels. For each AFM measurement, the spring constant of the cantilever was calibrated using thermal tuning method and it was found to be in the range of $0.035-0.045 \mathrm{~N} / \mathrm{m}$. Siliconnitride AFM probes with a four-sided pyramid geometry were used. For this specific type of probe, Equation 1 describes the Hertzian model used to determine the Young's modulus of the material $(E)$ based on the relation between force $(F)$ and indentation $(\delta)(6)$ : 


$$
F=\frac{1}{\sqrt{2}} \frac{E}{\left(1-v^{2}\right)} \tan (\alpha) \delta^{2}
$$

$\alpha$ stands for the half face angle of the probe, while $v$ stands for the Poisson's ratio (assumed to be 0.48 ).

All the recorded AFM images consisted of either $8 \times 8$ or $16 \times 16$ pixels with scan size varying from 10 to 50 $\mu \mathrm{m}$. Several AFM images were obtained at separate locations across the hydrogel surfaces to verify the reproducibility of the results. In order to account for hydrogel swelling, stiffness measurements were performed $24 \mathrm{~h}$ after specimen preparation and equilibration in PBS at $37^{\circ} \mathrm{C}$. Cell seeding also occurred after this $24 \mathrm{~h}$ swelling period.

\section{Cell culture}

Green fluorescence protein (GFP)-expressing HUVECs (Angioproteomie, Boston, MA) were cultured in medium EBM-2 (Lonza, Basel, Switzerland) supplemented with EGM-2 bullet kit (Lonza, Basel, Switzerland) at $37^{\circ} \mathrm{C}$ and $5 \% \mathrm{CO}_{2}$. Between 3000 and 4000 GFP-HUVEC cells per $\mathrm{cm}^{2}$ were seeded on polyacrylamide hydrogels with specific stiffness. Cells were allowed to attach and spread overnight before imaging. Gels were prepared with embedded fiducial markers for traction calculation and functionalized with either fibronectin or collagen for cell adhesion.

\section{Focal adhesion visualization}

A vinculin reporter, part of a tension FRET sensor (Vin-TS), was used to visualize focal adhesions. Vin-TS (plasmid \#26019, Addgene) was a gift from Martin Schwartz (7). HUVECs, passage 3-5, were seeded at approximately $70 \%$ confluency and incubated with transfection medium without heparin and antibiotics. After overnight attachment, cells were transfected with $1 \mu \mathrm{g} /$ well of the Vin-TS plasmids using XtremeGene HP DNA Transfection Reagent (Merck, Kenilworth, NJ, USA) according to the manufacturer's instructions. After a $2 \mathrm{~h}$ incubation, the transfection reagent was washed off; after which cells are expected to express the proteins for a minimum of 24 hours; cells were then reseeded as described in the previous section.

\section{Live cell imaging}

Imaging was done using an Olympus FluoView ${ }^{\mathrm{TM}}$ FV1000-IX81 (Olympus, Tokio, Japan) confocal microscope, equipped with an incubator controlling temperature and $\mathrm{CO}_{2}$, and a Leica HC FL FLUOTAR 40X/0.600 CORR long working distance objective (Leica, Wetzlar, Germany). Images of the cells and the stressed hydrogels (deformed under cellular tractions) were taken simultaneously every 2.25 min for approximately $1 \mathrm{~h}$ (67.5min or 30 time-points) using a $488 \mathrm{~nm}$ laser for GFP-HUVECs and a $559 \mathrm{~nm}$ laser for fluorescent beads. A Z-stack of depth between 5.6 and $7 \mu \mathrm{m}$ from the hydrogel surface was taken for each imaged cell to avoid possible loss of focus during the time-lapse. Image volumes were acquired with $0.4 \mu \mathrm{m}$ pixel size in the $\mathrm{XY}$ plane and $0.7 \mu \mathrm{m}$ along the Z-axis.

To obtain the reference gel configuration for TFM, cells were detached from the hydrogels at the end of the experiment by adding sodium dodecyl sulphate (SDS) $20 \%$ (Sigma-Aldrich, St. Louis, MO). The hydrogels were allowed to stabilize for some minutes and Z-stacks of the relaxed (undeformed) hydrogels were taken at corresponding locations where cells had been previously recorded. 
Prior to the calculation of hydrogel displacements, acquired bead images were filtered by a difference of Gaussians operator to simultaneously boost blob-like structures and reduce noise. Then, the XY plane representing the surface of the hydrogel was identified within the acquired Z-stack.

The calculation of the 2D displacement fields from bead images was split into two image registration steps. First, a global rigid registration of the images was performed to correct for the stage shifts that might be present during time-lapse acquisitions. Then, the bead images of the stressed hydrogel at each time point were compared to the bead images of the relaxed hydrogel acquired after cell lysis, and Free Form Deformation (FFD)-based non-rigid registration (8) was used to compute the cell-induced displacement field. To obtain smooth displacement fields and at the same time cope with different levels of matrix deformations, the FFD algorithm was applied following a coarse-to-fine multiscale strategy. Specifically, a three-level multiscale approach was defined with $72 \times 72$ pixels, $36 \times 36$ pixels, and $18 \times 18$ pixels as the size of the deformable mesh used by the FFD algorithm for the coarsest, intermediate, and finest scales, respectively.

Regularized traction recovery

PA hydrogel substrates were considered as homogeneous, isotropic, linear elastic half spaces, and cell tractions were recovered by Tikhonov regularized Fourier Transform Traction Cytometry algorithm (9) as:

$$
\boldsymbol{t}_{\lambda}=\mathcal{F}^{-1}\left\{\left(\widetilde{\boldsymbol{G}}^{*} \widetilde{\boldsymbol{G}}+\lambda \boldsymbol{I}\right)^{-1} \widetilde{\boldsymbol{G}}^{*} \widetilde{\boldsymbol{u}}\right\}
$$

where recovered cellular tractions $\boldsymbol{t}_{\lambda}$ are obtained as the inverse Fourier transform $\left(\mathcal{F}^{-1}\right)$ of the regularized inversion of the elasticity problem in the Fourier domain. Here, $\widetilde{\boldsymbol{G}}$ and $\widetilde{\boldsymbol{u}}$ represent the Green's function expressed as a tensor and the displacement field, respectively, in the Fourier domain, $\widetilde{\boldsymbol{G}}^{*}$ is the complex conjugate of $\widetilde{\boldsymbol{G}}$, and $\lambda$ is a parameter that controls the amount of regularization applied.

\section{Gel stiffness independent regularization}

While needed to prevent noise amplification and therefore to stabilize the solution in TFM experiments, Tikhonov regularization also acts as a smoothing operator. Hence, over-regularization (i.e. choosing a high $\lambda$ value) can lead to the underestimation of the recovered tractions, at the same time distributing them over larger areas. Given the impact of $\lambda$ on the recovered traction field, the amount of regularization must be kept constant when comparing tractions between different experimental conditions, such as different substrate stiffness values. However, since the Green's function is inversely proportional to the Young's modulus $(E)$ of the substrate, the amount of regularization will scale with $E^{2}$ for a constant value of $\lambda$. Indeed, using $\widetilde{\boldsymbol{G}}_{0}$ to denote the Green's function in the Fourier domain for $E=E_{0}=1$ and fixing the value of the regularization parameter to $\lambda=\lambda_{c}$, Equation 2 can be rewritten as:

$$
\begin{aligned}
\boldsymbol{t}_{\lambda_{c}} & =\mathcal{F}^{-1}\left\{\left(\frac{1}{E} \widetilde{\boldsymbol{G}}_{0}^{*} \frac{1}{E} \widetilde{\boldsymbol{G}}_{0}+\lambda_{c} \boldsymbol{I}\right)^{-1} \frac{1}{E} \widetilde{\boldsymbol{G}}_{0}^{*} \widetilde{\boldsymbol{u}}\right\} \\
& =E \mathcal{F}^{-1}\left\{\left(\widetilde{\boldsymbol{G}}_{0}^{*} \widetilde{\boldsymbol{G}}_{0}+E^{2} \lambda_{c} \boldsymbol{I}\right)^{-1} \widetilde{\boldsymbol{G}}_{0}^{*} \widetilde{\boldsymbol{u}}\right\}
\end{aligned}
$$


Equation 3 demonstrates that for a constant $\lambda_{c}$, larger penalties $E^{2} \lambda_{c} \boldsymbol{I}$ will be imposed for stiffer substrates. To overcome this unwanted effect, we reformulated the calculation of the cellular tractions as:

$$
\boldsymbol{t}_{\lambda_{c}}=E \boldsymbol{t}_{\lambda_{c}, E_{0}}
$$

with

$$
\boldsymbol{t}_{\lambda_{c}, E_{0}}=\mathcal{F}^{-1}\left\{\left(\widetilde{\boldsymbol{G}}_{0}^{*} \widetilde{\boldsymbol{G}}_{0}+\lambda_{c} \boldsymbol{I}\right)^{-1} \widetilde{\boldsymbol{G}}_{0}^{*} \widetilde{\boldsymbol{u}}\right\}
$$

being the regularized traction recovery for $E=E_{0}$. Hence, the obtained tractions were regularized independently of the Young's modulus, allowing for unbiased comparison among cells cultured on hydrogels of different rigidity. Video S1 shows a representative example of the effects of both noncorrected and corrected regularization applied to the same displacement field.

\section{Temporal tracking of traction foci}

After the traction foci were defined for cell images at each time-point, each focus was given an identifier (focus ID). A custom function was used to track individual foci in time (i.e. correlate foci IDs between consecutive time-points), ensuring that each traction focus had a unique focus ID for its entire lifetime within the experiment. The tracking algorithm was adapted from an algorithm to track 3D cellular clusters in time developed in part by one of the authors (10). The algorithm checked that there is at least a single pixel overlap between traction foci in consecutive time-steps as a requirement to assign the same focus ID. If two (or more) foci merged, the new focus was labeled as the larger of the two. Finally, if a traction focus shared no pixels with any focus in the previous time-point, this was considered a new focus and awarded a new focus ID. Alternatively, traction foci could cease to exist if no pixel were shared by any traction focus in the subsequent time-point; a traction focus was allowed to disappear for up to 3 timepoints (6.75 $\mathrm{min}$ ) before considered defunct.

\section{Supplemental Video Caption}

Video S1. Representative example of the effects of both non-corrected (a) and corrected (b) regularization applied to the same displacement field. (a) Note expansion of traction footprint in non-corrected case as value of Young's modulus is increased. (b) Note linear scaling of traction magnitude with value of Young's modulus in corrected case. Displacement field was measured for a HUVEC on hydrogel of $2.7 \mathrm{kPa}$ stiffness coated with collagen. Traction calculation was performed for multiple values of the hydrogel's Young's modulus (ranging between 1-100 kPa). Scale bar (30 $\mu \mathrm{m})$.

Video S2. Representative example of the distribution of focal adhesions along the cell-hydrogel interface. Vinculin fluorescent reporter used to image focal adhesions in a cell in time. Vinculin fluorescence is displayed in red, while the traction foci are outlined in white; white circles indicate the position of the local peaks in traction magnitude for each focus. Images were processed to make focal adhesions more visible: The contrast was enhanced followed by application of a top-hat filter to remove fluorescence of the nucleus. Vinculin intensity was imaged for a HUVEC cultured on a hydrogel of $2.7 \mathrm{kPa}$ stiffness coated with collagen. Scale bar $(30 \mu \mathrm{m})$.

Video S3. Position of focal adhesions relative to traction foci. Not all focal adhesions were detected (see Video S2), but strongest foci are always associated with large focal adhesions, and adhesions near the centroid of the cell shape do not exert 
tractions. Traction foci are colored according to their traction magnitude, while focal adhesions and cell outline are colored in pink. The video corresponds to the same cell in Video S2, a HUVEC cultured on a hydrogel of $2.7 \mathrm{kPa}$ stiffness coated with collagen. Scale bar $(30 \mu \mathrm{m})$.

\section{Supplemental Figures}
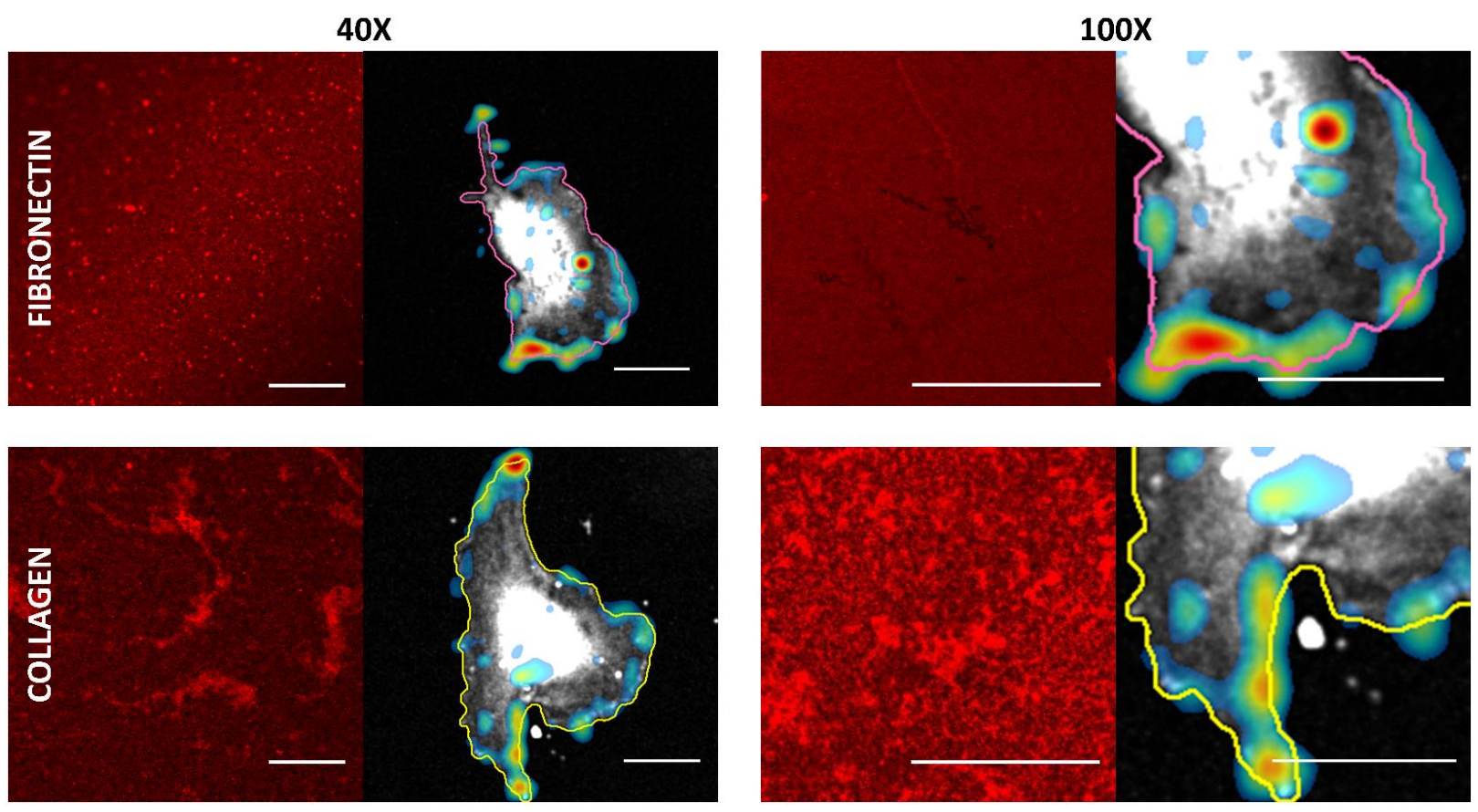

Figure S1. The cell binding area of polyacrylamide hydrogels is saturated with coating protein. Immunostaining of fibronectin (TOP) and collagen (BOTTOM) coating of polyacrylamide hydrogels acquired using objective lenses with two different magnifications: 40X (LEFT) and 100X (RIGHT). Immunostaining was performed in hydrogels synthesized and coated according to the procedure outlined in the Methods section; however, no cells were seeded in these gels. Cells shown, were grown on gels with the same treatment (i.e. intermediate stiffness of $2.7 \mathrm{kPa}$, and coated with the indicated protein coating), are used for comparison of the size of the traction foci with features of the protein coating. Image of GFP-HUVECs shown in grayscale, with traction footprint colored according to traction magnitude as well as cell outline in pink (cell cultured on fibronectin) or yellow (cell cultured on collagen). Scale bar $(30 \mu \mathrm{m})$. 


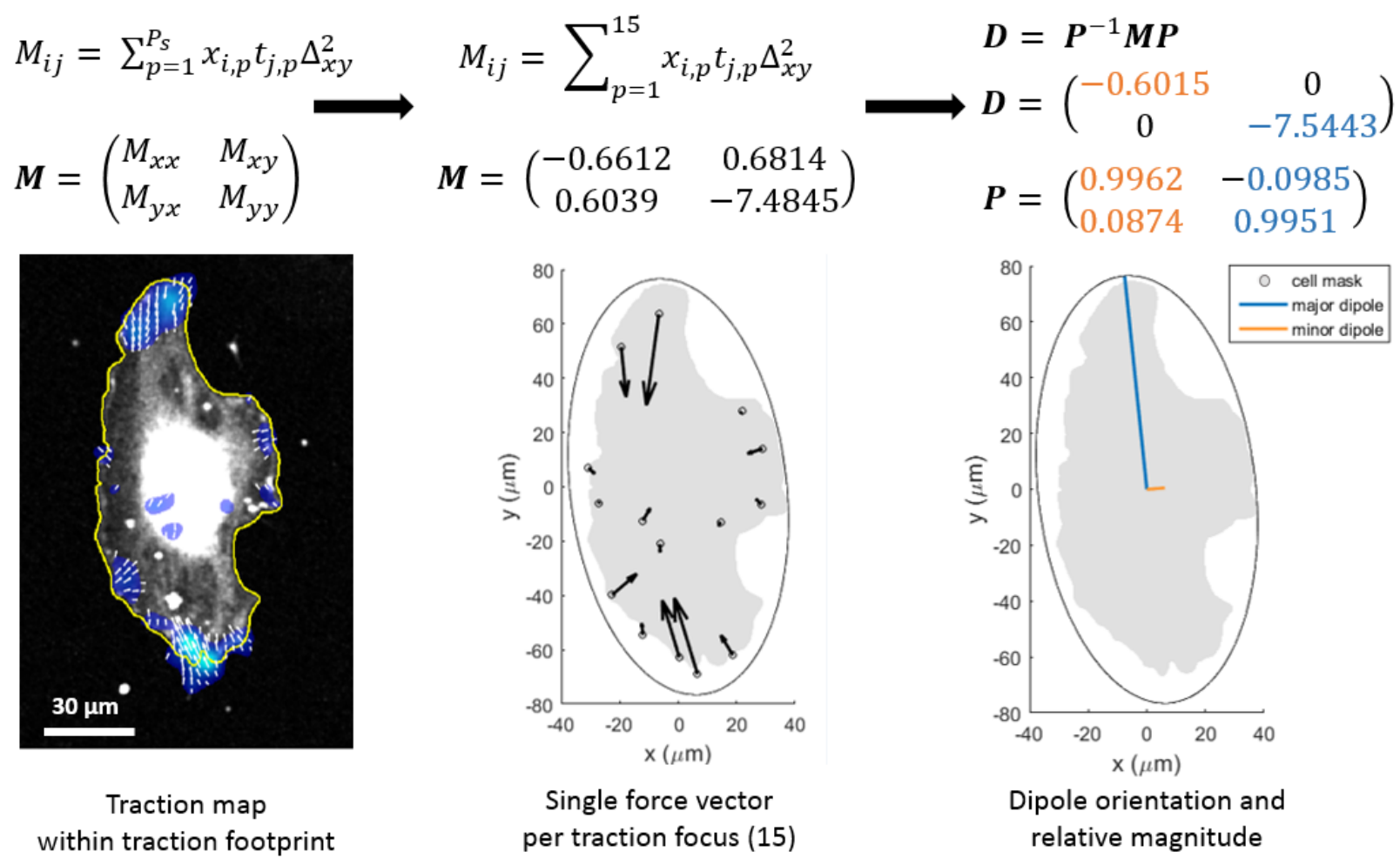

Figure S2. Sample calculation of the force dipole $(\boldsymbol{M})$ for a single time-point for a cell seeded on a poly acrylamide hydrogel of intermediate stiffness $(2.7 \mathrm{kPa}$ ) coated with collagen. (left) Image of GFP-HUVEC with overlaid traction footprint and cell perimeter. (center) Simplified tractions resulting from summing tractions within each traction focus and placed in position of peak value of focus: This was done only for displaying purposes, showing clearly the general orientation of the tractions at the cell-substrate interface. For all reported results, dipole was calculated by summing over all pixels in the traction footprint and maintaining the position of the tractions. (right) Diagonalization of $\boldsymbol{M}$, resulting in identification of major and minor dipole, displayed graphically in blue and orange respectively over the cell mask. Mathematical symbols: $M_{i j}$ represents the components of the dipole, where $i$ and $j$ refer to the coordinate axes $(x=1, y=2) ; x_{i, p}$ is the $i t h$ component of the position of each pixel $(p)$ from the centroid of the cell mask; $t_{j, p}$ is the $j t h$ component of the traction at the corresponding pixel; $\Delta_{x y}^{2}$ is the area of each pixel; $P_{S}$, is the number of pixels in the traction footprint. 


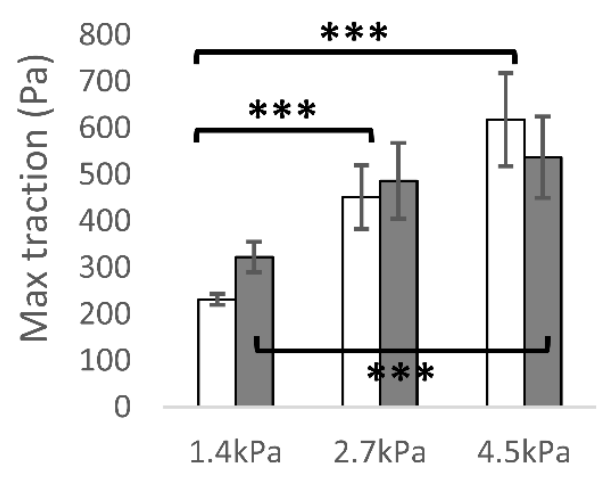

口FIBRONECTIN $\square$ COLLAGEN

Figure S3. Maximum tractions calculated for cells on different stiffness hydrogels functionalized with fibronectin or collagen. Average \pm SEM ( $n=15$ cells for 1.5 and $2.7 \mathrm{kPa}$ fibronectin, $n=12$ cells in $4.5 \mathrm{kPa}$ fibronectin and $1.5 \mathrm{kPa}$ collagen, $n=10$ cells for $2.7 \mathrm{kPa}$ collagen and $\mathrm{n}=11$ cells on $4.5 \mathrm{kPa}$ collagen). Statistical significance: $* \mathrm{p}<0.05, * * * \mathrm{p}<0.005$

a

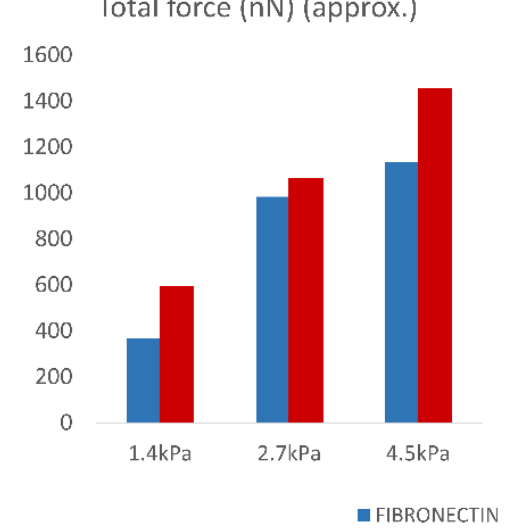

b

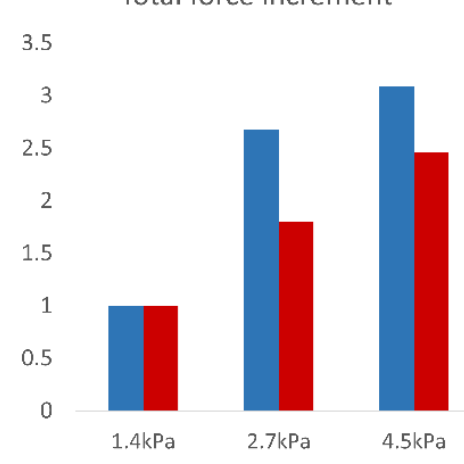

- Collagen

Figure S4. (a) Approximation of the total force exerted by cells in each condition throughout the duration of an experiment. Values obtained by multiplying the average cumulative number of foci observed (Figure $3 b$, Main Text) by the average force value exerted by foci (Figure 5c, Main Text). (b) Normalized values relative to low stiffness condition for each ECM protein. 
a

Area $\left(\mu \mathrm{m}^{2}\right)$

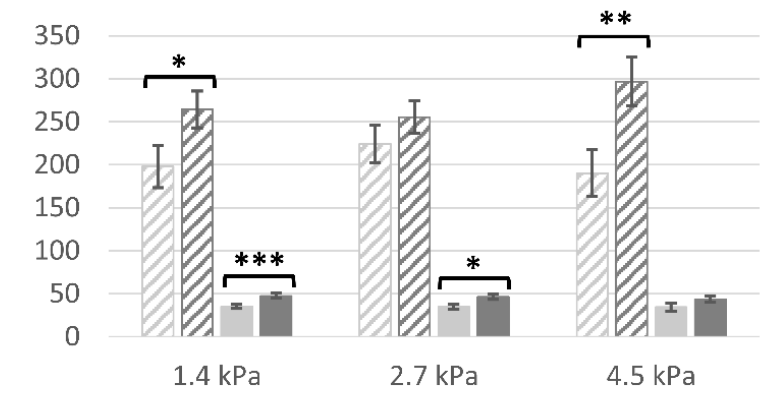

b

Force $(\mathrm{nN})$

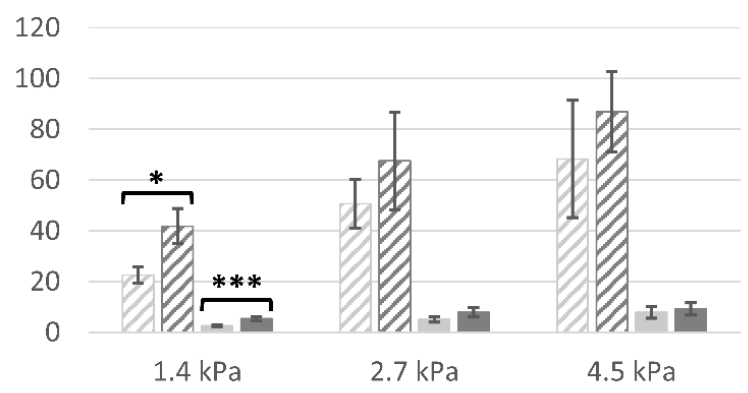

C

Mean focus traction (Pa)

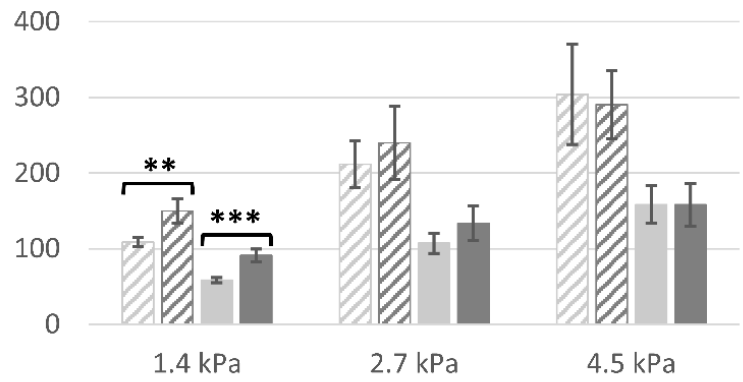

d

Lifetime (min)

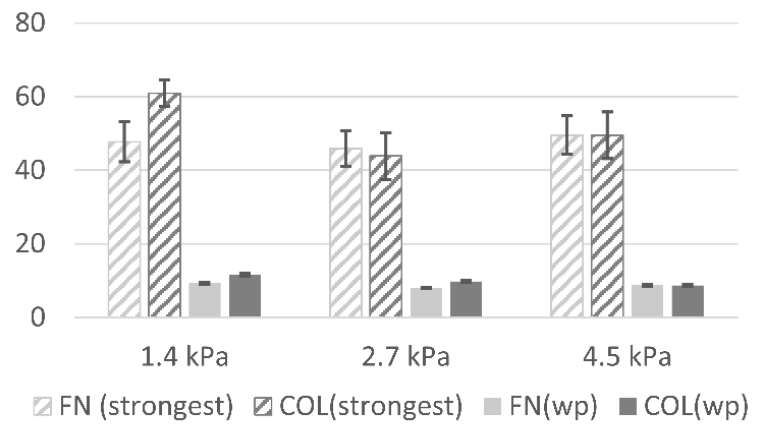

Figure S5. Characterization of whole and strongest populations of traction foci and comparison between use of fibronectin (FN) and collagen (COL) as ECM protein on the hydrogel. Values represent averages over all foci per experimental condition of: (a) Area; (b) Force; (c) Mean focus traction; (d) Lifetime. Average \pm SD were plotted. Abbreviations: collagen (COL), fibronectin (FN), whole population (wp). $p$-values represent the statistical difference between fibronectin and collagen for a single stiffness value: $* \mathrm{p}<0.05, * * \mathrm{p}<0.01, * * * \mathrm{p}<0.005$. 

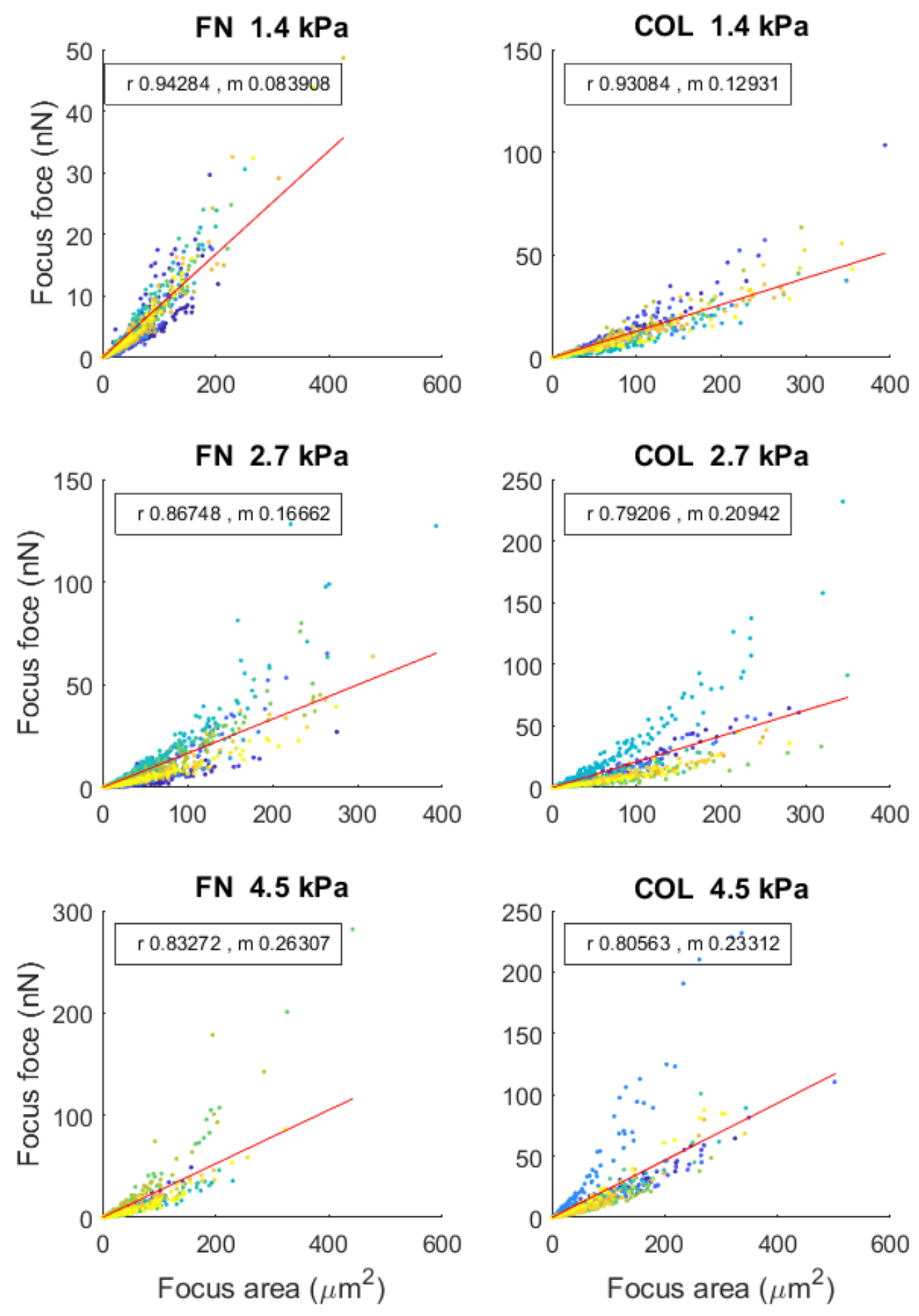

Figure S6. Scatter plot of traction foci area and traction foci force per condition. Each point represents the average over the entire lifetime of the focus. Different colors represent different cells. Legend: $r$ - correlation coefficient indicating linearity, $m$ - slope of linear fit. Abbreviations: collagen (COL), fibronectin (FN). 

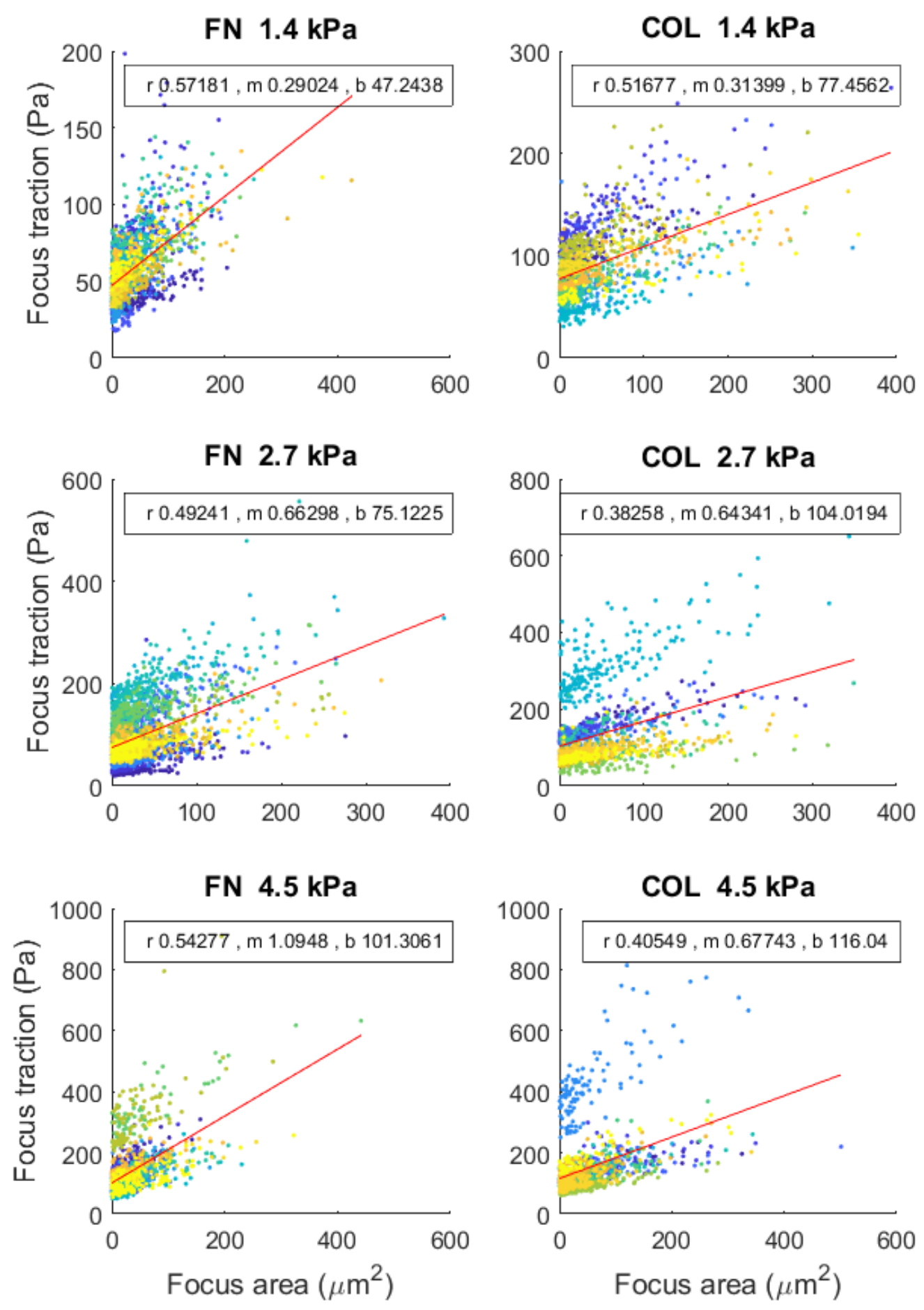

Figure S7. Scatter plot of traction foci area and traction foci average traction per condition. Each point represents the average over the entire lifetime of the focus. Different colors represent different cells. Legend: $r$ - correlation coefficient indicating linearity, $\mathrm{m}$ - slope of linear fit, $\mathrm{b}$ - vertical axis intercept. Abbreviations: collagen (COL), fibronectin (FN). 

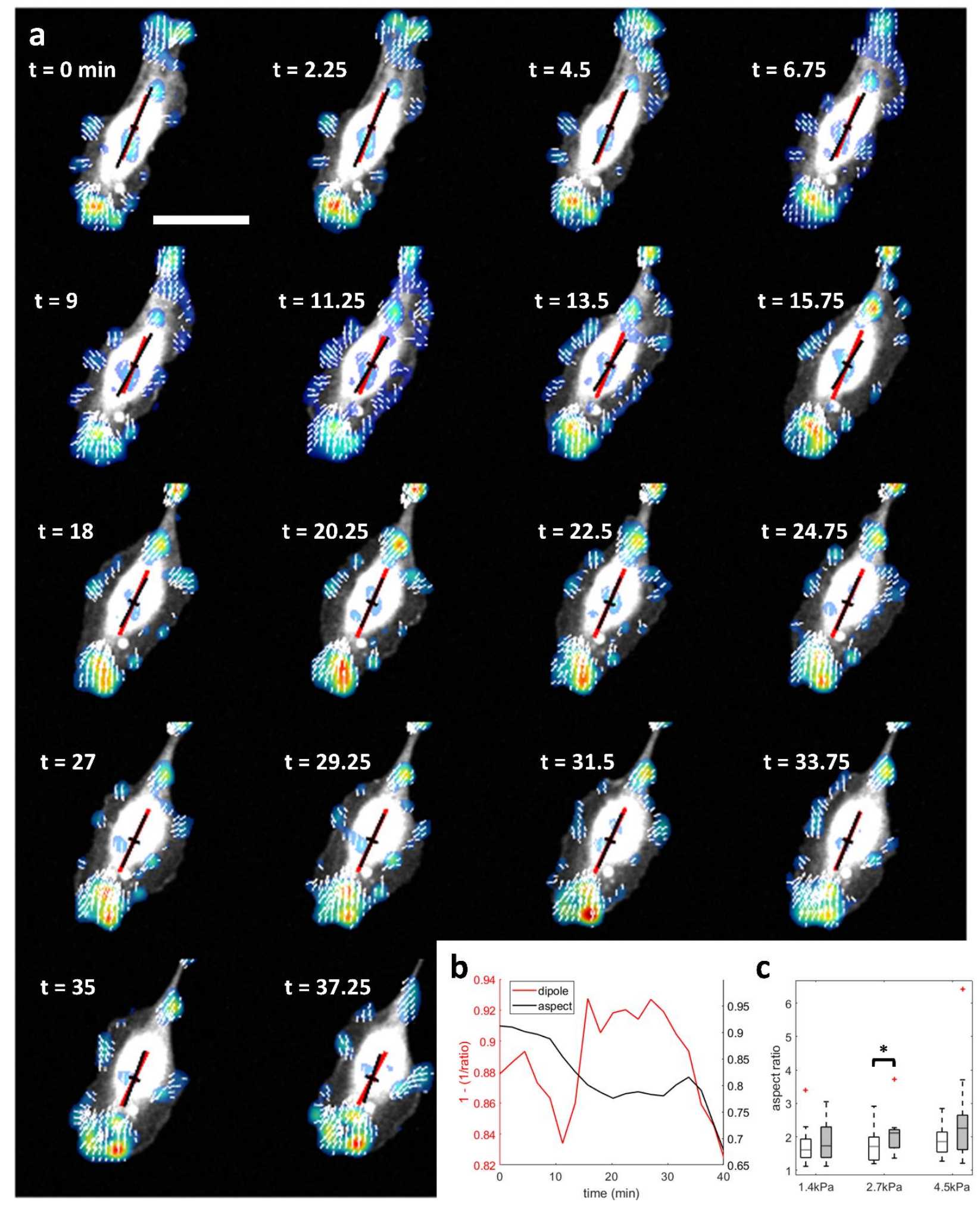

Figure S8. Morphology (aspect ratio) and force (dipole ratio) polarization are not always correlated. a) Brightfield images of HUVECs with a cytoplasmic dye with overlaid traction footprint (color indicates magnitude, arrows indicate direction), major and minor dipole orientations and relative size (red), and major and minor axes, which define the aspect ratio, based on cell mask (black). Cell is seeded on $2.7 \mathrm{kPa}$ hydrogel coated with collagen. b) Comparison of dipole and aspect ratios. Quantity plotted is $1-$ (1/ratio) such that it is normalized, and a higher values represent higher polarization. c) Time-averaged aspect ratio of cells on 
different stiffness hydrogels functionalized with fibronectin or collagen. Central mark indicates median. Top and bottom edges of box indicate first and third quartiles, respectively. Whiskers show extent of lowest and highest values. Outliers are marked by ' + ' symbol. ( $n=15$ cells for 1.5 and $2.7 \mathrm{kPa}$ fibronectin, $n=12$ cells in $4.5 \mathrm{kPa}$ fibronectin and $1.5 \mathrm{kPa}$ collagen, $n=10$ cells for 2.7 $\mathrm{kPa}$ collagen and $\mathrm{n}=11$ cells on $4.5 \mathrm{kPa}$ collagen). Statistical significance: $* p<0.05$

\section{Supplemental References}

1. Tse, J.R., and A.J. Engler. 2010. Preparation of hydrogel substrates with tunable mechanical properties. Curr. Protoc. Cell Biol. 47: 1-16.

2. Kraning-Rush, C.M., S.P. Carey, J.P. Califano, B.N. Smith, and C. a Reinhart-King. 2011. The role of the cytoskeleton in cellular force generation in 2D and 3D environments. Phys. Biol. 8: 15009.

3. Califano, J.P., and C.A. Reinhart-King. 2008. A Balance of Substrate Mechanics and Matrix Chemistry Regulates Endothelial Cell Network Assembly. Cell. Mol. Bioeng. 1: 122-132.

4. Rahman, A., S.P. Carey, C.M. Kraning-Rush, Z.E. Goldblatt, F. Bordeleau, M.C. Lampi, D.Y. Lin, A.J. García, and C.A. Reinhart-King. 2016. Vinculin regulates directionality and cell polarity in two- and three-dimensional matrix and three-dimensional microtrack migration. Mol. Biol. Cell. 27: 14311441.

5. Krishnan, R., D.D. Klumpers, C.Y. Park, K. Rajendran, X. Trepat, J. van Bezu, V.W.M. van Hinsbergh, C. V. Carman, J.D. Brain, J.J. Fredberg, J.P. Butler, and G.P. van Nieuw Amerongen. 2011. Substrate stiffening promotes endothelial monolayer disruption through enhanced physical forces. Am. J. Physiol. Physiol. 300: C146-C154.

6. Rico, F., P. Roca-Cusachs, N. Gavara, R. Farré, M. Rotger, and D. Navajas. 2005. Probing mechanical properties of living cells by atomic force microscopy with blunted pyramidal cantilever tips. Phys. Rev. E. 72: 21914.

7. Grashoff, C., B.D. Hoffman, M.D. Brenner, R. Zhou, M. Parsons, M.T. Yang, M.A. McLean, S.G. Sligar, C.S. Chen, T. Ha, and M.A. Schwartz. 2010. Measuring mechanical tension across vinculin reveals regulation of focal adhesion dynamics. Nature. 466: 263-6.

8. Jorge-Peñas, A., A. Izquierdo-Alvarez, R. Aguilar-Cuenca, M. Vicente-Manzanares, J.M. GarciaAznar, H. Van Oosterwyck, E.M. de-Juan-Pardo, C. Ortiz-de-Solorzano, and A. Muñoz-Barrutia. 2015. Free Form Deformation-Based Image Registration Improves Accuracy of Traction Force Microscopy. PLoS One. 10: e0144184.

9. Sabass, B., M.L. Gardel, C.M. Waterman, and U.S. Schwarz. 2008. High resolution traction force microscopy based on experimental and computational advances. Biophys. J. 94: 207-220.

10. Sharma, Y., D.A. Vargas, A.F. Pegoraro, D. Lepzelter, D.A. Weitz, and M.H. Zaman. 2015. Collective Motion of Mammalian Cell Cohorts in 3D. Integr. Biol. 7: 1526-1533. 